Lectures in FORMATION AND INTERACTIONS OF TOPOLOGICAL DEFECTS, NATO ASI B349, ed A.-C. Davis, R. Brandenberger, pp 303-348

(Plenum, New York, 1995).

\title{
DYNAMICS OF COSMIC STRINGS AND OTHER BRANE MODELS
}

\section{Brandon Carter.}

C.N.R.S., Départment d'Astrophysique Relativiste et de Cosmologie, Observatoire de Paris, 92195 Meudon, FRANCE.

\begin{abstract}
The supporting worldsheet of a string, membrane, or other higher dimensional brane, is analysed in terms of its first, second, and third fundamental tensors, and its inner and outer curvature tensors. The dynamical equations governing the models appropriate for phenomena such as (superconducting) cosmic strings and cosmic domain walls are developed in a general framework (allowing for both electromagnetic and Kalb Ramond background coupling). It is shown how the surface stress momentum energy density tensor determines the propagation characteristics of small "wiggle" perturbations of the worldsheet. Attention is then focussed on special features of strings (using the transonic model with tension $T$ inversely proportional to the energy density $U$ as a particularly important example). A quadratic Hamilton-Jacobi formulation is shown to govern equilibium states and other conservative string configurations sharing a symmetry of the (gravitational, electromagnetic, and Kalb-Ramond) background, including stable ring states that may be cosmologically important.
\end{abstract}

\section{Worldsheet Curvature Analysis}

In preparation for the more specific study of strings in the last three sections of this course, the first three sections are intended as an introduction to the systematic study, in a classical relativistic framework, of "branes", meaning physical models in which the relevant fields are confined to supporting worldsheets of lower dimension than the background spacetime. While not entirely new [1] 2], this subject is still at a rather early stage of development (compared with the corresponding quantum theory[3] which has been stimulated by the rise of "superstring theory"), the main motivation for recent work 1 on classical relativistic brane theory being its application to vacuum defects produced by the Kibble mechanism[5], particularly when of composite type as in the case of cosmic strings attached 10 external domain walls 6 ] and of cosmic strings carrying internal currents $[7]$.

Before discussing the dynamic laws governing the evolution of a brane worldsheet it is worthwhile to devote this first section to a recapitulation of the essential differential geometric machinery [8] [9] needed for the analysis of a timelike worldsheet of dimension d say in a background space time manifold of dimension n. At this stage no restriction will be imposed on the curvature of the metric - which will as usual be represented with respect to local background coordinates $x^{\mu}$ ( $\mu=$ $0, \ldots, \mathrm{n}-1)$ by its components $g_{\mu \nu}-$ though it will be postulated to be flat, or at least stationary 
string and membrane theory has been hampered by a tradition of publishing results in untidy, highly gauge dependent, notation (one of the causes being the undue influence still exercised by Eisenhart's obsolete treatise "Riemannian Geometry" [10]). For the intermediate steps in particular calculations it is of course frequently useful and often indispensible to introduce specifically adapted auxiliary structures, such as curvilinear worldsheet coordinates $\sigma^{i}(i=0, \ldots, \mathrm{d}-1)$ and the associated bitensorial derivatives

$$
x_{, i}^{\mu}=\frac{\partial x^{\mu}}{\partial \sigma^{i}},
$$

or specially adapted orthonormal frame vectors, consisting of an internal subset of vectors $\iota_{A}{ }^{\mu}$ ( $\mathrm{A}=0, \ldots, \mathrm{d}-1)$ tangential to the worldsheet and an external subset of vectors $\lambda_{X}{ }^{\mu}(\mathrm{X}=1, \ldots$, $\mathrm{n}-\mathrm{d}$ ) orthogonal to the worldsheet, as characterised by

$$
\iota_{A}{ }^{\mu} \iota_{B} \mu=\eta_{A B}, \quad \iota_{A}{ }^{\mu} \lambda_{X \mu}=0, \quad \lambda_{X}{ }^{\mu} \lambda_{Y \mu}=\delta_{X Y},
$$

where $\eta_{A B}$ is a fixed d-dimensional Minkowski metric and the Kronecker matrix $\delta_{X Y}$ is a fixed (n-d)-dimensional Cartesion metric. Even in the most recent literature there are still (under Eisenhart's uninspiring influence) many examples of insufficient effort to sort out the messy clutter of indices of different kinds (Greek or Latin, early or late, small or capital) that arise in this way by grouping the various contributions into simple tensorially covariant combinations. Another inconvenient feature of many publications is that results have been left in a form that depends on some particular gauge choice (such as the conformal gauge for internal string coordinates) which obscures the relationship with other results concerning the same system but in a different gauge.

The strategy adopted here[11] aims at minimising such problems (they can never be entirely eliminated) by working as far as possible with a single kind of tensor index, which must of course be the one that is most fundamental, namely that of the background coordinates, $x^{\mu}$. Thus, to avoid dependence on the internal frame index A (which is lowered and raised by contraction with the fixed d-dimensional Minkowski metric $\eta_{A B}$ and its inverse $\eta^{A B}$ ) and on the external frame index $\mathrm{X}$ (which is lowered and raised by contraction with the fixed (n-d)-dimensional Cartesian metric $\delta_{X Y}$ and its inverse $\left.\delta^{X Y}\right)$, the separate internal frame vectors $\iota_{A}{ }^{\mu}$ and external frame vectors $\lambda_{X}{ }^{\mu}$ will as far as possible be eliminated in favour of the frame gauge independent combinations

$$
\eta_{\nu}^{\mu}=\iota_{A}{ }^{\mu} \iota^{A}{ }_{\nu}, \quad \perp_{\nu}^{\mu}=\lambda_{X}{ }^{\mu} \lambda^{X}{ }_{\nu}
$$

of which the former, $\eta_{\nu}^{\mu}$, is what will be referred to as the (first) fundamental tensor of the metric, which acts as the (rank d) operator of tangential projection onto the world sheet, while the latter, $\perp_{\nu}^{\mu}$, is the complementary (rank $\mathrm{n}-\mathrm{d}$ ) operator of projection orthogonal to the world sheet.

The same principle applies to the avoidance of unnecessary involvement of the internal coordinate indices which are lowered and raised by contraction with the induced metric on the worldsheet as given by

$$
h_{i j}=g_{\mu \nu} x^{\mu}{ }_{, i} x^{\nu}{ }_{, j},
$$


and with its contravariant inverse $h^{i j}$. After being cast (by index raising if necessary) into its contravariant form, any internal coordinate tensor can be directly projected onto a corresponding background tensor in the manner exemplified by the intrinsic metric itself, which gives

$$
\eta^{\mu \nu}=h^{i j} x_{, i}^{\mu} x_{, j}^{\nu},
$$

thus providing an alternative (more direct) prescription for the fundamental tensor that was previously introduced via the use of the internal frame in (1.3). This approach also provides a direct prescription for the orthogonal projector that was introduced via the use of an external frame in (1.3) but that is also obtainable immediately from (1.5) as

$$
\perp_{\nu}^{\mu}=g_{\nu}^{\mu}-\eta_{\nu}^{\mu}
$$

In so far as we are concerned with tensor fields such as the frame vectors whose support is confined to the d-dimensional world sheet, the effect of Riemannian covariant differentation $\nabla_{\mu}$ along an arbitrary directions on the background spacetime will not be well defined, only the corresponding tangentially projected differentiation operation

$$
\bar{\nabla}_{\mu} \stackrel{\text { def }}{=} \eta^{\nu}{ }_{\mu} \nabla_{\nu}
$$

being meaningful for them, as for instance in the case of a scalar field $\varphi$ for which the tangentially projected gradient is given in terms of internal coordinate differentiation simply by $\bar{\nabla}^{\mu} \varphi=h^{i j} x^{\mu}{ }_{i} \varphi, j$.

An irreducible basis for the various possible covariant derivatives of the frame vectors consists of the internal rotation pseudo-tensor $\rho_{\mu}{ }_{\rho} \rho$ and the external rotation (or "twist") pseudo-tensor $\omega_{\mu \rho}^{\nu}$ as given by

$$
\rho_{\mu \rho}^{\nu}=\eta_{\sigma}^{\nu} \iota^{A}{ }_{\rho} \bar{\nabla}_{\mu} \iota_{A}{ }^{\sigma}=-\rho_{\mu \rho}{ }^{\nu}, \quad \omega_{\mu}{ }^{\nu}{ }^{\nu}=\perp^{\nu}{ }_{\sigma} \lambda^{x}{ }_{\rho} \bar{\nabla}_{\mu} \lambda_{X}{ }^{\sigma}=-\omega_{\mu \rho}{ }^{\nu},
$$

together with the second fundamental tensor $K_{\mu \nu}{ }^{\rho}$ as given by

$$
K_{\mu \nu}{ }^{\rho}=\perp_{\sigma}^{\rho} \iota^{A}{ }_{\nu} \bar{\nabla}_{\mu} \iota_{A}{ }^{\sigma}=-\eta_{\nu}^{\sigma}{ }_{\nu}{ }_{X}{ }^{\rho} \bar{\nabla}_{\mu} \lambda^{X}{ }_{\sigma} .
$$

The reason for qualifying the fields (1.8) as "pseudo" tensors is that although they are tensorial in the ordinary sense with respect to changes of the background coordinates $x^{\mu}$ they are not geometrically well defined just by the geometry of the world sheet but are gauge dependent in the sense of being functions of the choice of the internal and external frames $\iota_{A}{ }^{\mu}$ and $\lambda_{X}{ }^{\mu}$. On the other hand, like the first fundamental tensor $\eta^{\mu \nu}$ as given by (1.5), the second fundamental tensor (1.9) is geometrically well defined in the sense of being frame gauge independent, as can be seen from the equivalent but more direct definition [4]

$$
K_{\mu \nu} \stackrel{\text { def }}{=} \eta_{\nu}^{\sigma}{ }_{\nu} \bar{\nabla}_{\mu} \eta_{\sigma}^{\rho}
$$


The gauge dependence of the rotation tensors $\rho_{\mu}{ }^{\nu}$ and $\omega_{\mu \rho}{ }^{\nu}$ means that (unlike $K_{\mu \nu}{ }^{\rho}$ ) they can each be set to zero at any particular given point on the worldsheet by choice of the relevant frames in its vicinity. However the condition for it to be possible to set these pseudo-tensors to zero throughout an open neigbourhood is the vanishing of the curvatures of the corresponding frame bundles as characterised with respect to the respective invariance subgroups $\mathrm{SO}(1, \mathrm{~d}-1)$ and $\mathrm{SO}(\mathrm{n}-\mathrm{d})$ into which the full Lorentz invariance group $\mathrm{SO}(1, \mathrm{n}-1)$ is broken by the specification of the d-dimensional world sheet orientation. The inner curvature that needs to vanish for it to be possible for $\rho_{\mu}{ }^{\nu}$ p to be set to zero in an open neighbourhood is of Riemannian type, is obtainable (by a calculation of the type originally developed by Cartan that was made familiar to physicists by Yang Mills theory) as [8]

$$
R_{\kappa \lambda}{ }^{\mu}{ }_{\nu}=2 \eta^{\mu}{ }_{\sigma} \eta^{\tau}{ }_{\mu} \eta^{\pi}{ }_{[\lambda} \bar{\nabla}_{\kappa]} \rho_{\pi}^{\sigma}{ }^{\sigma}+2 \rho_{[\kappa}{ }^{\mu \pi} \rho_{\lambda] \pi \nu},
$$

while the outer curvature that needs to vanish for it to be possible for the "twist" tensor $\omega_{\mu}{ }^{\nu}$ to be set to zero in an open neighbourhood is of a less familiar type that is given by

$$
\Omega_{\kappa \lambda}{ }_{\nu}^{\mu}=2 \perp_{\sigma}^{\mu} \perp_{\mu}^{\tau} \eta_{[\lambda}^{\pi} \bar{\nabla}_{\kappa]} \omega_{\pi \tau}^{\sigma}+2 \omega_{[\kappa}^{\mu \pi} \omega_{\lambda] \pi \nu} .
$$

The frame gauge invariance of the expressions (1.11) and (1.12) is not immediately obvious, but will be made manifest in the the alternative expressions to be given below following a synopsis of the properties of the (by (1.10) manifestly) gauge invariant second fundamental tensor $K_{\mu \nu}{ }^{\rho}$.

An equation of the form (1.10) for $K_{\mu \nu}{ }^{\rho}$ is of course meaningful not only for the fundamental projection tensor of a d-surface, but also for any (smooth) field of rank-d projection operators $\eta^{\mu}{ }_{\nu}$ as specified by a field of arbitrarily orientated d-surface elements. What distinguishes the integrable case, i.e. that in which the elements mesh together to form a well defined d-surface through the point under consideration, is the condition that the tensor defined by (1.10) should also satisfy the Weingarten identity

$$
K_{[\mu \nu]}^{\rho}=0
$$

(where the square brackets denote antisymmetrisation), this symmetry property of the second fundamental tensor being derivable [4] as a version of the well known Frobenius theorem. In addition to this non-trivial symmetry property, the second fundamental tensor is also obviously tangential on the first two indices and almost as obviously orthogonal on the last, i.e.

$$
\perp_{\mu}^{\sigma} K_{\sigma \nu}{ }^{\rho}=K_{\mu \nu}{ }^{\sigma} \eta_{\sigma}{ }^{\rho}=0 .
$$

The second fundamental tensor $K_{\mu \nu}{ }^{\rho}$ has the property of fully determining the tangential derivatives of the first fundamental tensor $\eta^{\mu}{ }_{\nu}$ by the formula

$$
\bar{\nabla}_{\mu} \eta_{\nu \rho}=2 K_{\mu(\nu \rho)}
$$


(using round brackets to denote symmetrisation) and it can be seen to be characterisable by the condition that the orthogonal projection of the acceleration of any tangential vector field $u^{\mu}$ will be given by

$$
u^{\mu} u^{\nu} K_{\mu \nu}^{\rho}=\perp_{\mu}^{\rho} \dot{u}^{\mu}, \quad \dot{u}^{\mu}=u^{\nu} \nabla_{\nu} u^{\mu}
$$

It is very practical for a great many purposes to introduce the extrinsic curvature vector $K^{\mu}$, defined as the trace of the second fundamental tensor, which is automatically orthogonal to the worldsheet,

$$
K^{\mu} \stackrel{\text { def }}{=} K_{\nu}^{\nu}{ }_{\nu}^{\mu}, \quad \eta_{\nu}^{\mu} K^{\nu}=0
$$

It is useful for many specific purposes to work this out in terms of the intrinsic metric $h_{i j}$ and its determinant $|h|$. It suffices to use the simple expression $\bar{\nabla}^{\mu} \varphi=h^{i j} x^{\mu}{ }_{, i} \varphi, j$ for the tangentially projected gradient of a scalar field $\varphi$ on the worldsheet, but for a tensorial field (unless one is using Minkowski coordinates in a flat spacetime) there will also be contributions involving the background Riemann Christoffel connection

$$
\Gamma_{\mu \rho}^{\nu}=g^{\nu \sigma}\left(g_{\sigma(\mu, \rho)}-\frac{1}{2} g_{\mu \rho, \sigma}\right) .
$$

The curvature vector is thus obtained in explicit detail as

$$
K^{\nu}=\bar{\nabla}_{\mu} \eta^{\mu \nu}=\frac{1}{\sqrt{\|h\|}}\left(\sqrt{\|h\|} h^{i j} x_{, i}^{\nu}\right), j+h^{i j} x_{, i}^{\mu} x_{, j}{ }_{, j} \Gamma_{\mu}{ }^{\nu} \rho .
$$

This last expression is technically useful for certain specific computational purposes, but it must be remarked that much of the literature on cosmic string dynamics has been made unnecessarily heavy to read by a tradition of working all the time with long strings of non tensorial terms such as those on the right of (1.19) rather than taking advantage of such more succinct tensorial expressions as the preceeding formula $\bar{\nabla}_{\mu} \eta^{\mu \nu}$. As an alternative to the universally applicable tensorial approach advocated here, there is of course another more commonly used method of achieving succinctness in particular circumstances, which is to sacrifice gauge covariance by using specialised kinds of coordinate system. In particular for the case of a string, i.e. for a 2-dimensional worldsheet, it is standard practise to use conformal coordinates $\sigma^{0}$ and $\sigma^{1}$ so that the corresponding tangent vectors $\dot{x}^{\mu}=x_{, 0}^{\mu}$ and $x^{\prime \mu}=x_{, 1}^{\mu}$ satisfy the restrictions $\dot{x}^{\mu} x^{\prime}{ }_{\mu}=0, \dot{x}^{\mu} \dot{x}_{\mu}+x^{\prime \mu} x_{\mu}{ }_{\mu}=0$, which implies $\sqrt{\|h\|}=x^{\prime \mu} x_{\mu}^{\prime}=-\dot{x}^{\mu} \dot{x}_{\mu}$ so that (1.19) simply gives $\sqrt{\|h\|} K^{\nu}=x^{\prime \prime \nu}-\ddot{x}^{\nu}+\left(x^{\prime \mu} x^{\prime \rho}-\dot{x}^{\mu} \dot{x}^{\rho}\right) \Gamma_{\mu}^{\nu}$.

The physical specification of the extrinsic curvature vector (1.17) for a timelike d-surface in a dynamic theory provides what can be taken as the equations of extrinsic motion of the $\mathrm{d}$ surface[4][9], the simplest possibility being the "harmonic" condition $K^{\mu}=0$ that is obtained (as will be shown in the following sections) from a surface measure variational principle such as that of the Dirac membrane model[1], or of the Goto-Nambu string model[5] whose dynamic equations in a flat background are therefore expressible with respect to a standard conformal gauge in the familiar form $x^{\prime \prime \mu}-\ddot{x}^{\mu}=0$. There is a certain analogy between the Einstein vacuum 
equations, which impose the vanishing of the trace $\mathcal{R}_{\mu \nu}$ of the background spacetime curvature $\mathcal{R}_{\lambda \mu}{ }^{\rho}{ }_{\nu}$, and the Dirac-Gotu-Nambu equations, which impose the vanishing of the trace $K^{\nu}$ of the second fundamental tensor $K_{\lambda \mu}{ }^{\nu}$. Just as it is useful to separate out the Weyl tensor[12], i.e. the trace free part of the Ricci background curvature which is the only part that remains when the Einstein vacuum equations are satisfied, so also analogously, it is useful to separate out the the trace free part of the second fundamental tensor, namely the extrinsic conformation tensor [8], which is the only part that remains when equations of motion of the Dirac-Gotu-Nambu type are satisfied. Explicitly, the trace free extrinsic conformation tensor $C_{\mu \nu}{ }^{\rho}$ of a d-dimensional imbedding is defined[8] in terms of the corresponding first and second fundamental tensors $\eta_{\mu \nu}$ and $K_{\mu \nu}{ }^{\rho}$ as

$$
C_{\mu \nu} \stackrel{\text { def }}{=} K_{\mu \nu}{ }^{\rho}-\frac{1}{\mathrm{~d}} \eta_{\mu \nu} K^{\rho}, \quad C_{\nu}^{\nu}{ }^{\mu}=0 .
$$

Like the Weyl tensor $\mathcal{W}_{\lambda \mu}{ }^{\rho}{ }_{\nu}$ of the background metric (whose definition is given implicitly by (1.25) below) this conformation tensor has the noteworthy property of being invariant with respect to conformal modifications of the background metric:

$$
g_{\mu \nu} \mapsto \mathrm{e}^{2 \alpha} g_{\mu \nu}, \quad \Rightarrow \quad K_{\mu \nu}{ }^{\rho} \mapsto K_{\mu \nu}{ }^{\rho}+\eta_{\mu \nu} \perp^{\rho \sigma} \nabla_{\sigma} \alpha, \quad C_{\mu \nu}{ }^{\rho} \mapsto C_{\mu \nu}{ }^{\rho} .
$$

This formula is useful 13 for calculations of the kind undertaken by Vilenkin 14 in a standard Robertson-Walker type cosmological background, which can be obtained from a flat auxiliary spacetime metric by a conformal transformation for which $\mathrm{e}^{\alpha}$ is a time dependent Hubble expansion factor.

As the higher order analogue of (1.10) we can go on to introduce the third fundamental tensor [4] as

$$
\Xi_{\lambda \mu \nu} \stackrel{\text { def }}{=} \eta_{\mu}^{\sigma}{ }_{\mu} \eta_{\nu}^{\tau} \perp_{\alpha}^{\rho} \bar{\nabla}_{\lambda} K_{\sigma \tau}{ }^{\alpha}
$$

which by construction is obviously symmetric between the second and third indices and tangential on all the first three indices. In a spacetime background that is flat (or of constant curvature as is the case for the DeSitter universe model) this third fundamental tensor is fully symmetric over all the first three indices by what is interpretable as the generalised Codazzi identity which is expressible [8] in a background with arbitrary Riemann curvature $\mathcal{R}_{\lambda \mu}{ }^{\rho}{ }_{\sigma}$ as

$$
\Xi_{\lambda \mu \nu}{ }^{\rho}=\Xi_{(\lambda \mu \nu)}^{\rho}+\frac{2}{3} \eta^{\sigma}{ }_{\lambda} \eta^{\tau}{ }_{(\mu} \eta_{\nu)}^{\alpha} \mathcal{R}_{\sigma \tau}{ }^{\beta}{ }_{\alpha} \perp_{\beta}{ }_{\beta}
$$

It is to be noted that a script symbol $\mathcal{R}$ is used here in order to distinguish the (n-dimensional) background Riemann curvature tensor from the intrinsic curvature tensor (1.11) of the (d-dimensional) worldship to which the ordinary symbol $R$ has already allocated.

For many of the applications that will follow it will be sufficient just to treat the background spacetime as flat, i.e. to take $\mathcal{R}_{\sigma \tau}{ }^{\beta}{ }_{\alpha}=0$. At this stage however, we shall allow for an unrestricted background curvature. For $\mathrm{n}>2$ this will be decomposible in terms of its trace free Weyl part 
$\mathcal{W}_{\mu \nu}{ }^{\rho}{ }_{\sigma} 5$ which as remarked above is conformally invariant) and the corresponding background Ricci tensor and its scalar trace,

$$
\mathcal{R}_{\mu \nu}=\mathcal{R}_{\rho \mu}{ }^{\rho}{ }_{\nu}, \quad \mathcal{R}=\mathcal{R}^{\nu}{ }_{\nu},
$$

in the form 12

$$
\mathcal{R}_{\mu \nu}^{\rho \sigma}=\mathcal{W}_{\mu \nu}^{\rho \sigma}+\frac{4}{n-2} g_{[\mu}^{[\rho} \mathcal{R}_{\nu]}^{\sigma]}-\frac{2}{(n-1)(n-2)} \mathcal{R} g_{[\mu}^{[\rho} g_{\nu]}^{\sigma]},
$$

(in which the Weyl contribution can be non zero only for $\mathrm{n} \geq 4$ ). In terms of the tangential projection of this background curvature, one can evaluate the corresponding internal curvature tensor (1.11) in the form

$$
R_{\mu \nu}{ }_{\sigma}{ }_{\sigma}=2 K^{\rho}{ }_{[\mu}{ }^{\tau} K_{\nu] \sigma \tau}+\eta^{\kappa}{ }_{\mu} \eta^{\lambda}{ }_{\nu} \mathcal{R}_{\kappa \lambda}{ }^{\alpha}{ }_{\tau} \eta^{\rho}{ }_{\alpha} \eta^{\tau}{ }_{\sigma},
$$

which is the translation into the present scheme of what is well known in other schemes as the generalised Gauss identity. The much less well known analogue for the (identically trace free and conformally invariant) outer curvature (1.12) (for which the most historically appropriate name might be argued to be that of Schouten[12]) is given [8] in terms of the corresponding projection of the background Weyl tensor by the expression

$$
\Omega_{\mu \nu}{ }^{\rho}{ }_{\sigma}=2 C_{[\mu}{ }^{\tau \rho} C_{\nu] \tau \sigma}+\eta^{\kappa}{ }_{\mu} \eta^{\lambda}{ }_{\nu} \mathcal{W}_{\kappa \lambda}{ }^{\alpha}{ }_{\tau} \perp_{\alpha}^{\rho}{ }_{\alpha}{ }_{\sigma}^{\tau}
$$

It follows from this last identity that in a background that is flat or conformally flat (for which it is necessary, and for $\mathrm{n} \geq 4$ sufficient, that the Weyl tensor should vanish) the vanishing of the extrinsic conformation tensor $C_{\mu \nu}{ }^{\rho}$ will be sufficient (independently of the behaviour of the extrinsic curvature vector $\left.K^{\mu}\right)$ for vanishing of the outer curvature tensor $\Omega_{\mu \nu}{ }^{\rho}{ }_{\sigma}$, which is the condition for it to be possible to construct fields of vectors $\lambda^{\mu}$ orthogonal to the surface and such as to satisfy the generalised Fermi-Walker propagation condition to the effect that $\perp_{\mu}{ }_{\mu} \bar{\nabla}_{\nu} \lambda_{\rho}$ should vanish. It can also be shown [8] (taking special trouble for the case $\mathrm{d}=3$ ) that in a conformally flat background (of arbitrary dimension n) the vanishing of the conformation tensor $C_{\mu \nu}{ }^{\rho}$ is always sufficient (though by no means necessary) for conformal flatness of the induced geometry in the imbedding.

The application with which we shall mainly be concerned in the following work will be the case $\mathrm{d}=2$ of a string. An orthonormal tangent frame will consist in this case just of a timelike unit vector, $\iota_{0}{ }^{\mu}$, and a spacelike unit vector, $\iota_{1}^{\mu}$, whose exterior product vector is the frame independent antisymmetric unit surface element tensor

$$
\mathcal{E}^{\mu \nu}=2 \iota_{0}{ }^{[\mu} \iota_{1}{ }^{\nu]}=2(-|h|)^{-1 / 2} x^{[\mu}{ }_{0} x^{\nu]}{ }_{, 1},
$$

whose tangential gradient satisfies

$$
\bar{\nabla}_{\lambda} \mathcal{E}^{\mu \nu}=-2 K_{\lambda \rho}{ }^{[\mu} \mathcal{E}^{\nu] \rho}
$$


(This is the special $\mathrm{d}=2$ case of what is, as far as I am aware, the only wrongly printed formula in the more complete analysis [8] on which this section is based: the relevant general formula (B9) is valid as printed only for odd $d$, but needs insertion of a missing sign adjustment factor $(-1)^{\mathrm{d}-1}$ in order to hold for all d.) In this case the inner rotation pseudo tensor (1.8) is determined just by a corresponding rotation covector $\rho_{\mu}$ according to the specification

$$
\rho_{\lambda}^{\mu}{ }_{\nu}=\frac{1}{2} \mathcal{E}^{\mu}{ }_{\nu} \rho_{\lambda}, \quad \rho_{\lambda}=\rho_{\lambda}{ }_{\lambda}{ }_{\nu} \mathcal{E}^{\nu}{ }_{\mu} .
$$

This can be used to see from (1.11) that the Ricci scalar (1.24) of the 2-dimensional worldsheet will have the well known property of being a pure surface divergence, albeit of a frame gauge dependent quantity:

$$
R=\bar{\nabla}_{\mu}\left(\mathcal{E}^{\mu \nu} \rho_{\nu}\right) .
$$

In the specially important case of a string in ordinary 4-dimensional spacetime, i.e. when we have not only $\mathrm{d}=2$ but also $\mathrm{n}=4$, the antisymmetric background measure tensor $\varepsilon^{\lambda \mu \nu \rho}$ can be used to determine a scalar (or more strictly, since its sign is orientation dependent, a pseudo scalar) magnitude $\Omega$ for the outer curvature tensor (1.12) (despite the fact that its traces are identically zero) according to the specification

$$
\Omega=\frac{1}{2} \Omega_{\lambda \mu \nu \rho} \varepsilon^{\lambda \mu \nu \rho}
$$

Under these circumstances one can also define a "twist" covector $\omega_{\mu}$, that is the outer analogue of $\rho_{\mu}$, according to the specification

$$
\omega_{\nu}=\frac{1}{2} \omega_{\nu}^{\mu \lambda} \varepsilon_{\lambda \mu \rho \sigma} \mathcal{E}^{\rho \sigma}
$$

This can be used to deduce from (1.12) that the outer curvature (pseudo) scalar $\Omega$ of a string worldsheet in 4-dimensions has a divergence property of the same kind as that of its more widely known Ricci analogue (1.31), the corresponding formula being given by

$$
\Omega=\bar{\nabla}_{\mu}\left(\mathcal{E}^{\mu \nu} \omega_{\nu}\right)
$$

It is to be remarked that for a compact spacelike 2-surface the integral of (1.29) gives the well known Gauss Bonnet invariant, but that the timelike string worldsheets under consideration here will not be characterised by any such global invariant since they will not be compact (being open in the time direction even for a loop that is closed in the spacial sense). The outer analogue of the Gauss Bonnet invariant that arises from (1.32) for a spacelike 2-surface has been discussed by Penrose and Rindler 15 but again there is no corresponding global invariant in the necessarily non-compact timelike case of a string worldsheet. 


\section{Laws of Motion for a Regular Brane Complex}

The term p-brane has come into use [3] [16] to describe a dynamic system localised on a timelike support surface of dimension $\mathrm{d}=\mathrm{p}+1$, imbedded in a spacetime background of dimension $\mathrm{n}>\mathrm{p}$. Thus at the low dimensional extreme one has the example of a zero - brane, meaning what is commonly referred to as a "point particle", and of a 1-brane meaning what is commonly referred to as a "string". At the high dimensional extreme one has the "improper" case of an (n-1)brane, meaning what is commonly referred to as a "medium" (as exemplified by a simple fluid), and of an (n-2)-brane, meaning what is commonly referred to as a "membrane" (from which the generic term "brane" is derived). A membrane (as exemplified by a cosmological domain wall) has the special feature of being supported by a hypersurface, and so being able to form a boundary between separate background space time regions; this means that a 2-brane has the status of being a membrane in ordinary 4-dimensional spacetime (with $n=4$ ) but not in a higher dimensional (e.g. Kaluza Klein type) background.

The purpose of the present section is to consider the dynamics not just of an individual brane but of a brane complex or "rigging model" $\mid$ such as is illustrated by the nautical archetype in which the wind - a 3-brane - acts on a boat's sail - a 2-brane - that is held in place by cords 1-branes - which meet at knots, shackles and pulley blocks that are macroscopically describable as point particles - i.e. 0-branes. In order for a a set of branes of diverse dimensions to qualify as a "geometrically regular" brane complex or "rigging system" it is required not only that the support surface of each (d-1)-brane should be a smoothly imbedded d-dimensional timelike hypersurface but also that its boundary, if any, should consist of a disjoint union of support surfaces of an attatched subset of lower dimensional branes of the complex. (For example in order qualify as part of a regular brane complex the edge of a boat's sail can not be allowed to flap freely but must be attatched to a hem cord belonging to the complex.) For the brane complex to qualify as regular in the strong dynamic sense that will be postulated in the present work, it is also required that a member p-brane can exert a direct force only on an an attached (p-1)-brane on its boundary or on an attached $(\mathrm{p}+1)$-brane on whose boundary it is itself located, though it may be passively subject to forces exerted by a higher dimensional background field. For instance the Peccei-Quin axion model gives rise to field configurations representable as regular complexes of domain walls attached to strings 17] 18] 19, and a bounded (topological or other) Higgs vortex defect terminated by a pair of pole defects 20] 212 223] may be represented as a regular brane complex consisting of a finite cosmic string with a pair of point particles at its ends, in an approximation neglecting Higgs field radiation. (However allowance for radiation would require the use of an extended complex including the Higgs medium whose interaction with the string - and a fortiori with the terminating particles - would violate the regularity condition: the ensuing singularities in the back reaction would need to be treated by a renormalisation procedure of a kind 24 25 that is beyond the scope of the present article, but that is discussed by Paul Shellard in an accompanying article in this 
volume and elsewhere[18].)

The present section will be restricted to the case of a brane complex that is not only regular in the sense of the preceeding paragraph but that is also pure (or "fine") in the sense that the lengthscales characterising the internal structure of the (defect or other) localised phenomenon represented by the brane models are short compared with those characterising the macroscopic variations under consideration so that polarisation effects play no role. For instance in the case of a point particle, the restriction that it should be describable as a "pure" zero brane simply means that it can be represented as a simple monopole without any dipole or higher multipole effects. In the case of a cosmic string the use of a "pure" 1-brane description requires that the underlying vortex defect be sufficiently thin compared not only compared with its total length but also compared with the lengthscales characterising its curvature and the gradients of any currents it may be carrying. The effect of the simplest kind of curvature corrections beyond this "pure brane" limit will be discussed in Section 3, but in the rest of this article, as in the present section, it will be assumed that the ratio of microscopic to macroscopic lengthscales is sufficiently small for description in terms of "pure" p-branes to be adequate.

The present section will not be concerned with the specific details of particular cases but with the generally valid laws that can be derived as Noether identities from the postulate that the model is governed by dynamical laws derivable from a variational principle specified in terms of an action function $\mathcal{I}$. It is however to be emphasised that the validity at a macroscopic level of the laws given here is not restricted to cases represented by macroscopic models of the strictly conservative type directly governed by a macroscopic variational principle. The laws obtained here will also be applicable to classical models of dissipative type (e.g. allowing for resistivity to relative flow by internal currents) as necessary conditions for the existence of an underlying variational description of the microscopic (quantum) degrees of freedom that are allowed for merely as entropy in the macroscopically averaged classical description.

In the case of a brane complex, the total action $\mathcal{I}$ will be given as a sum of distinct d-surface integrals respectively contributed by the various $(\mathrm{d}-1)$-branes of the complex, of which each is supposed to have its own corresponding Lagrangian surface density scalar (d) $\mathcal{L}$ say. Each supporting d-surface will be specified by a mapping $\sigma \mapsto x\{\sigma\}$ giving the local background coordinates $x^{\mu}$ $(\mu=0, \ldots, \mathrm{n}-1)$ as functions of local internal coordinates $\sigma^{i}(\mathrm{i}=0, \ldots, \mathrm{d}-1)$. The corresponding $\mathrm{d}-$ dimensional surface metric tensor (d) $h_{i j}$ that is induced (in the manner described in the preceeding section) as the pull back of the n-dimensional background spacetime metric $g_{\mu \nu}$, will determine the natural surface measure, (d) $\overline{\mathcal{S}}$, in terms of which the total action will be expressible in the form

$$
\mathcal{I}=\sum_{\mathrm{d}} \int^{(\mathrm{d})} \bar{d}^{(\mathrm{S})} \overline{\mathcal{L}}, \quad{ }^{(\mathrm{d})} \overline{d \mathcal{S}}=\sqrt{\|(\mathrm{d}) h\|} d^{\mathrm{d}} \sigma .
$$

As a formal artifice whose use is an unnecessary complication in ordinary dynamical calculations but that can be useful for purposes such as the calculation of radiation, the confined (d-surface 
supported) but locally regular Lagrangian scalar fields (d) $\overline{\mathcal{L}}$ can be replaced by corresponding unconfined, so no longer regular but distributional fields (d) $\hat{\mathcal{L}}$, in order to allow the the basic multidimensional action (2.1) to be represented as a single integral,

$$
\mathcal{I}=\int d \mathcal{S} \sum_{\mathrm{d}}(\mathrm{d}) \hat{\mathcal{L}}, \quad d \mathcal{S}=\sqrt{\|g\|} d^{\mathrm{n}} x
$$

over the n-dimensional background spacetime. In order to do this, it is evident that for each (d-1)brane of the complex the required distributional action contribution (d) $\hat{\mathcal{L}}$ must be constructed in terms of the corresponding regular d-surface density scalar (d) $\overline{\mathcal{L}}$ according to the prescription that is expressible in standard Dirac notation as

$$
\text { (d) } \hat{\mathcal{L}}=\|g\|^{-1 / 2} \int{ }^{(\mathrm{d})} \overline{\mathcal{S}}(\mathrm{d}) \overline{\mathcal{L}} \delta^{\mathrm{n}}[x-x\{\sigma\}] .
$$

In the kind of model under consideration, each supporting d-surface is supposed to be endowed with its own independent internal field variables which are allowed to couple with each other and with their derivatives in the corresponding d-surface Lagrangian contribution (d) $\overline{\mathcal{L}}$, and which are also allowed to couple into the Lagrangian contribution $(\mathrm{d}-1) \overline{\mathcal{L}}$ on any of its attached boundary (d-1) surfaces, though - in order not to violate the strong dynamic regularity condition - they are not allowed to couple into contributions of dimension $(\mathrm{d}-2)$ or lower. As well as involving its own d-brane surface fields and those of any $(d+1)$ brane to whose boundary it may belong, each contribution (d) $\overline{\mathcal{L}}$ may also depend passively on the fields of a fixed higher dimensional background. Such fields will of course always include the background spacetime metric $g_{\mu \nu}$ itself. Apart from that, the most commonly relevant kind of backround field (the only one allowed for in the previous analysis[4]) is a Maxwellian gauge potential $A_{\mu}$ whose exterior derivative is the automatically "closed" electromagnetic field,

$$
F_{\mu \nu}=2 \nabla_{[\mu} A_{\nu]}, \quad \nabla_{[\mu} F_{\nu \rho]}=0 .
$$

Although many other possibilities can in principle be enviseaged, for the sake of simplicity the following analysis will not go beyond allowance for the only one that is important in applications to the kind of cosmic or superfluid defects that are the subject of discussion in the present volume, namely an antisymmetric Kalb-Ramond gauge field $B_{\mu \nu}=-B_{\nu \mu}$ whose exterior derivative is an automativally closed physical current 3-form,

$$
N_{\mu \nu \rho}=3 \nabla_{[\mu} B_{\nu \rho]}, \quad \nabla_{[\mu} N_{\nu \rho \sigma]}=0 .
$$

Just as a Maxwellian gauge transformation of the form $A_{\mu} \mapsto A_{\mu}+\nabla_{\mu} \alpha$ for an arbitrary scalar $\alpha$ leaves the electromagnetic field (2.4) invariant, so analogously a Kalb-Ramond gauge transformation $B_{\mu \nu} \mapsto B_{\mu \nu}+2 \nabla_{[\mu} \chi_{\nu]}$ for an arbitrary covector $\chi_{\mu}$ leaves the corresponding current 3-form 
(5) invariant. In applications to ordinary 4-dimensional spacetime, the current 3-form will just be the dual $N_{\mu \nu \rho}=\epsilon_{\mu \nu \rho \sigma} N^{\sigma}$ of an ordinary current vector $N^{\mu}$ satisfying a conservation law of the usual type, $\nabla_{\mu} N^{\mu}=0$. Such a Kalb-Ramond representation can be used to provide an elegant variational formulation for ordinary perfect fluid theory 26] and is particularly convenient for setting up "global" string models of vortices both in a simple cosmic axion or Higgs field 27 28] 29] and in a superfluid 30] such as liquid Helium-4.

In accordance with the preceeding considerations, the analysis that follows will be based on the postulate that the action is covariantly and gauge invariantly determined by specifying each scalar Lagrangian contribution (d) $\overline{\mathcal{L}}$ as a function just of the background fields, $A_{\mu}, B_{\mu \nu}$ and of course $g_{\mu \nu}$, and of any relevant internal fields (which in the simplest non-trivial case - exemplified by Witten[7 type superconducting string models 31 32] - consist just of a phase scalar $\varphi$ ). In accordance with the restriction that the branes be "pure" or "fine" in the sense explained above, it is postulated that polarisation effects are excluded by ruling out couplings involving gradients of the background fields. This means that the effect of making arbitrary infinitesimal "Lagrangian" variations $d_{\mathrm{L}} A_{\mu}, d_{\mathrm{L}} B_{\mu \nu}, d_{\mathrm{L}} g_{\mu \nu}$ of the background fields will be to induce a corresponding variation $d \mathcal{I}$ of the action that simply has the form

$$
d \mathcal{I}=\sum_{\mathrm{d}} \int^{(\mathrm{d})} \bar{d}\left\{{ }^{(\mathrm{d})} \bar{J}^{\mu} d_{\mathrm{L}} A_{\mu}+\frac{1}{2}{ }^{(\mathrm{d})} \bar{W}^{\mu \nu} d_{\mathrm{L}} B_{\mu \nu}+\frac{1}{2}{ }^{(\mathrm{d})} \bar{T}^{\mu \nu} d_{\mathrm{L}} g_{\mu \nu}\right\}
$$

provided either that that the relevant independent internal field components are fixed or else that the internal dynamic equations of motion are satisfied in accordance with the variational principle stipulating that variations of the relevant independent field variables should make no difference. For each d-brane of the complex, this partial differentiation formula implicitly specifies the corresponding electromagnetic surface current density vector (d) $\bar{J}^{\mu}$, the surface vorticity flux bivector ${ }^{(\mathrm{d})} \bar{W}^{\mu \nu}=-{ }_{(\mathrm{d})} \bar{W}^{\nu \mu}$, and the surface stress momentum energy density tensor ${ }^{(\mathrm{d})} \bar{T}^{\mu \nu}=$ (d) $\bar{T}^{\nu \mu}$, which are formally expressible more explicitly as

$$
{ }^{(\mathrm{d})} \bar{J}^{\mu}=\frac{\delta^{(\mathrm{d})} \overline{\mathcal{L}}}{\delta A_{\mu}}, \quad{ }^{(\mathrm{d})} \bar{W}^{\mu \nu}=2 \frac{\delta^{(\mathrm{d})} \overline{\mathcal{L}}}{\delta B_{\mu \nu}}, \quad{ }^{(\mathrm{d})} \bar{T}^{\mu \nu}=2 \frac{\delta^{(\mathrm{d})} \overline{\mathcal{L}}}{\delta g_{\mu \nu}}+{ }^{(\mathrm{d})} \overline{\mathcal{L}}^{(\mathrm{d})} \eta^{\mu \nu},
$$

where ${ }^{(\mathrm{d})} \eta^{\mu \nu}$ is the rank-d fundamental tensor of the d-dimensional imbedding, as defined in the manner described in the preceeding section.

The condition that the action be gauge invariant means that if one simply sets $d_{\mathrm{L}} A_{\mu}=\nabla_{\mu} \alpha$, $d_{\mathrm{L}} B_{\mu \nu}=2 \nabla_{[\mu} \chi_{\nu]}, d_{\mathrm{L}} g_{\mu \nu}=0$, for arbitrarily chosen $\alpha$ and $\chi_{\mu}$ then $d \mathcal{I}$ should simply vanish, i.e.

$$
\sum_{\mathrm{d}} \int d^{(\mathrm{d})} \overline{\mathcal{S}}\left\{{ }^{(\mathrm{d})} \bar{J}^{\mu} \nabla_{\mu} \alpha+{ }^{(\mathrm{d})} \bar{W}^{\mu \nu} \nabla_{\mu} \chi_{\nu}\right\}=0
$$

In order for this to be able to hold for all possible fields $\alpha$ and $\chi_{\mu}$ it is evident that the surface current ${ }^{(\mathrm{d})} \bar{J}^{\mu}$ and the vorticity flux bivector (d) $\bar{W}^{\mu \nu}$ must (as one would anyway expect from 
the consideration that they depend just on the relevant internal d-surface fields) be purely dsurface tangential, i.e. their contractions with the relevant rank (n-d) orthogonal projector (d) $\perp{ }_{\nu}^{\mu}=$ $g^{\mu}{ }_{\nu}-(\mathrm{d}) \eta^{\mu}{ }_{\nu}$ must vanish:

$$
\text { (d) } \perp_{\nu}^{\mu}{ }_{\nu}^{(\mathrm{d})} \bar{J}^{\nu}=0, \quad \text { (d) } \perp_{\nu}^{\mu}{ }_{\nu}^{(\mathrm{d})} \bar{W}^{\nu \rho}=0 .
$$

Hence, decomposing the full gradient operator $\nabla_{\mu}$ as the sum of its tangentially projected part ${ }^{(\mathrm{d})} \bar{\nabla}_{\mu}={ }^{(\mathrm{d})} \eta_{\mu}^{\nu} \nabla_{\nu}$ and of its orthogonally projected part ${ }^{\text {(d) } \perp_{\mu}^{\nu}}{ }_{\mu} \nabla_{\mu}$, and noting that by (2.9) the latter will give no contribution, one sees that (2.8) will take the form

$$
\sum_{\mathrm{d}} \int{ }^{(\mathrm{d})} \bar{d}\left\{{ }^{(\mathrm{d})} \bar{\nabla}_{\mu}\left({ }^{(\mathrm{d})} \bar{J}^{\mu} \alpha+{ }^{(\mathrm{d})} \bar{W}^{\mu \nu} \chi_{\nu}\right)-\alpha^{(\mathrm{d})} \bar{\nabla}_{\mu} \bar{J}^{\mu}-\chi_{\nu}{ }^{(\mathrm{d})} \bar{\nabla}_{\mu}{ }^{(\mathrm{d})} \bar{W}^{\mu \nu}\right\}=0,
$$

in which first term of each integrand is a pure surface divergence. Such a divergence can be dealt with using Green's theorem, according to which, for any d-dimensional support surface (d) $\overline{\mathcal{S}}$ of a (d-1)-brane, one has the identity

$$
\int^{(\mathrm{d})} \overline{\mathcal{S}}^{(\mathrm{d})} \bar{\nabla}_{\mu}{ }^{(\mathrm{d})} \overline{\boldsymbol{J}}^{\mu}=\oint^{(\mathrm{d}-1)} \overline{\boldsymbol{S}}^{(\mathrm{d})} \lambda_{\mu}{ }^{(\mathrm{d})} \bar{J}^{\mu}
$$

where the integral on the right is taken over the boundary (d-1)-surface of $\partial$ (d) $\overline{\mathcal{S}}$ of (d) $\overline{\mathcal{S}}$, and (d) $\lambda_{\mu}$ is the (uniquely defined) unit tangent vector on the d-surface that is directed normally outwards at its (d-1)-dimensional boundary. Bearing in mind that a membrane support hypersurface can belong to the boundary of two distinct media, and that for $\mathrm{d} \leq \mathrm{n}-3$ a d-brane may belong to a common boundary joining three or more distinct $(\mathrm{d}+1)$-branes of the complex under consideration, one sees that $(2.10)$ is equivalent to the condition

$$
\sum_{\mathrm{p}} \int^{(\mathrm{p})} \overline{\mathcal{S}}\left\{\alpha\left({ }^{(\mathrm{p})} \bar{\nabla}_{\mu}{ }^{(\mathrm{p})} \bar{J}^{\mu}-\sum_{\mathrm{d}=\mathrm{p}+1}{ }^{(\mathrm{d})} \lambda_{\mu}{ }^{(\mathrm{d})} \bar{J}^{\mu}\right)+\chi_{\nu}\left({ }^{(\mathrm{p})} \bar{\nabla}_{\mu}{ }^{(\mathrm{p})} \bar{W}^{\mu \nu}-\sum_{\mathrm{d}=\mathrm{p}+1}{ }^{(\mathrm{d})} \lambda_{\mu}{ }^{(\mathrm{d})} \bar{W}^{\mu \nu}\right)\right\}=0,
$$

where, for a particular p-dimensionally supported ( $\mathrm{p}-1$ )-brane, the summation "over $\mathrm{d}=\mathrm{p}+1$ " is to be understood as consisting of a contribution from each $(\mathrm{p}+1)$-dimensionally supported p-brane attached to it, where for each such p-brane, (d) $\lambda_{\mu}$ denotes the (uniquely defined) unit tangent vector on its $(p+1)$-dimensional support surface that is directed normally towards the p-dimensional support surface of the boundary ( $\mathrm{p}-1)$-brane. The Maxwell gauge invariance requirement to the effect that (2.12) should hold for arbitrary $\alpha$ can be seen to entail an electromagnetic charge conservation law of the form

$$
{ }^{(\mathrm{p})} \bar{\nabla}_{\mu}{ }^{(\mathrm{p})} \bar{J}^{\mu}=\sum_{\mathrm{d}=\mathrm{p}+1}{ }^{(\mathrm{d})} \lambda_{\mu}{ }^{(\mathrm{d})} \bar{J}^{\mu} .
$$

This can be seen from (2.11) to be be interpretable as meaning that the total charge flowing out of particular ( $\mathrm{d}-1)$-brane from its boundary is balanced by the total charge flowing into it 
from any d-branes to which it may be attached. The analogous Kalb-Ramond gauge invariance requirement that (2.12) should also hold for arbitrary $\chi_{\mu}$ can be seen to entail a corresponding vorticity conservation law of the form

$$
{ }^{(\mathrm{p})} \bar{\nabla}_{\mu}{ }^{(\mathrm{p})} \bar{W}^{\mu \nu}=\sum_{\mathrm{d}=\mathrm{p}+1}{ }^{(\mathrm{d})} \lambda_{\mu}{ }^{(\mathrm{d})} \bar{W}^{\mu \nu} .
$$

A more sophisticated but less practical way of deriving the foregoing conservation laws would be to work not from the expression (1) in terms of ordinary surface integrals but instead to use the superficially simpler expression (2.2) in terms of distributions which leads to the replacement of (2.13) by the ultimately equivalent (more formally obvious but less directly meaningful) expression

$$
\nabla_{\mu}\left(\sum_{\mathrm{d}}^{(\mathrm{d})} \hat{J}^{\mu}\right)=0
$$

involving the no longer regular but Dirac distributional current (d) $\hat{J}^{\mu}$ that is given in terms of the corresponding regular surface current $(\mathrm{d}) \bar{J}^{\mu}$ by

$$
\text { (d) } \hat{J}^{\mu}=\|g\|^{-1 / 2} \int(\mathrm{d}) \overline{\mathcal{S}}(\mathrm{d}) \bar{J}^{\mu} \delta^{\mathrm{n}}[x-x\{\sigma\}] .
$$

Similarly one can if one wishes rewrite the vorticity flux conservation law (14) in the distributional form

$$
\nabla_{\mu}\left(\sum^{(\mathrm{d})} \hat{W}^{\mu \nu}\right)=0
$$

where the distributional vorticity flux (d) $\hat{W}^{\mu \nu}$ is given in terms of the corresponding regular surface flux ${ }^{(\mathrm{d})} \bar{W}^{\mu \nu}$ by

$$
\text { (d) } \hat{W}^{\mu \nu}=\|g\|^{-1 / 2} \int{ }^{(\mathrm{d})} \overline{\mathcal{S}}{ }^{(\mathrm{d})} \bar{W}^{\mu \nu} \delta^{\mathrm{n}}[x-x\{\sigma\}] .
$$

It is left as an entirely optional exercise for any readers who may be adept in distribution theory to show how the ordinary functional relationships (2.13) and (2.14) can be recovered by by integrating out the Dirac distributions in (2.15) and(2.17).

The condition that the hypothetical variations introduced in (2.6) should be "Lagrangian" simply means that they are to be understood to be measured with respect to a reference system that is comoving with the various branes under consideration, so that their localisation with respect to it remains fixed. This condition is necessary for the variation to be meaningly definable at all for a field whose support is confined to a particular brane locus, but in the case of an unrestricted background field one can enviseage the alternative possibility of an "Eulerian" variation, meaning one defined with respect to a reference system that is fixed in advance, independently of the localisation of the brane complex, the standard example being that of a Minkowski reference system in the case of a background that is flat. In such a case the relation between the more generally meaningfull Lagrangian (comoving) variation, denoted by $d_{\mathrm{L}}$, and the corresponding 
Eulerian (fixed point) variation denoted by $d_{\mathrm{E}}$ say will be given by Lie differentiation with respect to the vector field $\xi^{\mu}$ say that specifies the infinitesimal of the comoving reference system with respect to the fixed background, i.e. one has

$$
d_{\mathrm{L}}-d_{\mathrm{E}}=\overrightarrow{\xi \mathcal{E}}
$$

where the Lie differentiation operator $\overrightarrow{\xi \mathcal{E}}$ is given for the background fields under consideration here by

$$
\begin{aligned}
& \overrightarrow{\xi \mathcal{E}} A_{\mu}=\xi^{\nu} \nabla_{\nu} A_{\mu}+A_{\nu} \nabla_{\mu} \xi^{\nu}, \\
& \overrightarrow{\xi \mathcal{E}} B_{\mu \nu}=\xi^{\nu} \nabla_{\nu} B_{\mu \nu}+2 B_{\rho[\nu} \nabla_{\mu]} \xi^{\rho}, \\
& \overrightarrow{\xi \in} g_{\mu \nu}=2 \nabla_{(\mu} \xi_{\nu)} .
\end{aligned}
$$

This brings us to the main point of this section which is the derivation of the dynamic equations governing the extrinsic motion of the branes of the complex, which are obtained from the variational principle to the effect that the action $\mathcal{I}$ is left invariant not only by infinitesimal variations of the relevant independent intrinsic fields on the support suerfaces but also by infinitesimal displacements of the support surfaces themselves. Since the background fields $A_{\mu}, B_{\mu \nu}$, and $g_{\mu \nu}$ are to be considered as fixed, the relevant Eulerian variations simply vanish, and so the resulting Lagrangian variations will be directly identifiable with the corresponding Lie derivatives - as given by (2.20) with respect to the generating vector field $\xi^{\mu}$ of the infinitesimal displacement under consideration. The variational principle governing the equations of extrinsic motion is thus obtained by setting to zero the result of substituting these Lie derivatives in place of the corresponding Lagrangian variations in the more general variation formula (2.6), which gives

$$
\sum_{\mathrm{d}} \int{ }^{(\mathrm{d})} \bar{d}\left\{{ }^{(\mathrm{d})} \bar{J}^{\mu} \overrightarrow{\xi \mathcal{E}} A_{\mu}+\frac{1}{2}{ }^{(\mathrm{d})} \bar{W}^{\mu \nu} \overrightarrow{\xi \mathcal{E}} B_{\mu \nu}+\frac{1}{2}{ }^{(\mathrm{d})} \bar{T}^{\mu \nu} \vec{\xi} \hat{\mathcal{L}} g_{\mu \nu}\right\}=0 .
$$

The requirement that this should hold for any choice of $\xi^{\mu}$ evidently implies that the tangentiality conditions (2.9) for the surface fluxes ${ }^{(\mathrm{d})} \bar{J}^{\mu}$ and ${ }^{(\mathrm{d})} \bar{W}^{\mu \nu}$ must be supplemented by an analogous dsurface tangentiality condition for the surface stress momentum energy tensor ${ }^{(\mathrm{d})} \bar{T}^{\mu \nu}$, which must satisfy

$$
\text { (d) } \perp_{\nu}^{\mu}(\mathrm{d}) \bar{T}^{\nu \rho}=0 .
$$

(as again one would expect anyway from the consideration that it depends just on the relevant internal d-surface fields). This allows (2.20) to be written out in the form

$$
\begin{aligned}
& \sum_{\mathrm{d}} \int{ }^{(\mathrm{d})} \bar{d}\left\{\xi^{\rho}\left(F_{\rho \mu}{ }^{(\mathrm{d})} \bar{J}^{\mu}+\frac{1}{2} N_{\rho \mu \nu}{ }^{(\mathrm{d})} \bar{W}^{\mu \nu}-{ }^{(\mathrm{d})} \bar{\nabla}_{\mu}{ }^{(\mathrm{d})} \bar{T}_{\rho}^{\mu}-A_{\rho}{ }^{(\mathrm{d})} \bar{\nabla}_{\mu}{ }^{(\mathrm{d})} \bar{J}^{\mu}-B_{\rho \nu}{ }^{(\mathrm{d})} \bar{\nabla}_{\mu}{ }^{(\mathrm{d})} \bar{W}^{\mu \nu}\right)\right. \\
& \left.{ }^{\left({ }^{(\mathrm{d})}\right.} \bar{\nabla}_{\mu}\left(\xi^{\rho}\left(A_{\rho}{ }^{(\mathrm{d})} \bar{J}^{\mu}+B_{\rho \nu}{ }^{(\mathrm{d})} \bar{W}^{\mu \nu}+{ }^{(\mathrm{d})} \bar{T}_{\rho}^{\mu}\right)\right)\right\}=0
\end{aligned}
$$


in which the final contribution is a pure surface divergence that can be dealt with using Green's theorem as before. Using the results (2.13) and (2.14) of the analysis of the consequences of gauge invariance and proceeding as in their derivation above, one sees that the condition for (2.23) to hold for an arbitrary field $\xi^{\mu}$ is that, on each (p-1)-brane of the complex, the dynamical equations

$$
{ }^{(\mathrm{p})} \bar{\nabla}_{\mu}{ }^{(\mathrm{p})} \bar{T}_{\rho}^{\mu}={ }^{(\mathrm{p})} f_{\rho}, \quad{ }^{(\mathrm{p})} f_{\rho}={ }^{(\mathrm{p})} \bar{f}_{\rho}+{ }^{(\mathrm{p})} \check{f}_{\rho}
$$

should be satisfied, where the contact force density $(\mathrm{p}) \check{f}_{\rho}$ exerted on the p-surface by other members of the brane complex is expressible as

$$
\text { (p) } \check{f}_{\rho}=\sum_{\mathrm{d}=\mathrm{p}+1}{ }^{(\mathrm{d})} \lambda_{\mu}{ }^{(\mathrm{d})} \bar{T}_{\rho}^{\mu},
$$

while the surface force density $(\mathrm{p}) \bar{f}_{\rho}$ exerted by the background fields is given by

$$
{ }^{(\mathrm{p})} \bar{f}_{\rho}=F_{\rho \mu}{ }^{(\mathrm{p})} \bar{J}^{\mu}+\frac{1}{2} N_{\rho \mu \nu}{ }^{(\mathrm{p})} \bar{W}^{\mu \nu} .
$$

As before the summation "over $\mathrm{d}=\mathrm{p}+1$ " in $(2.25)$ is to be understood as consisting of a contribution from each of the p-branes attached to the (p-1)- brane under consideration, where for each such attached p-brane, (d) $\lambda_{\mu}$ denotes the (uniquely defined) unit tangent vector on its $(\mathrm{p}+1)$ dimensional support surface that is directed normally towards the p-dimensional support surface of the boundary ( $\mathrm{p}-1)$-brane. The first of the background force contibutions in (2.26) is of course the Lorentz type force density resulting from the effect of the electromagnetic field on the surface current, while the other contribution in (2.26) is a Joukowsky type force density (of the kind responsible for the lift on an aerofoil) resulting from the Magnus effect, which acts in the case of a "global" string 27 28] though not in the case of a string of the "local" type for which the relevant

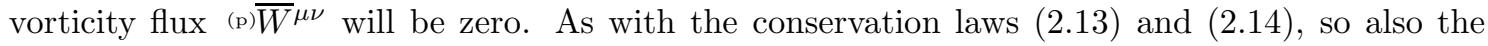
explicit force density balance law expressed by (2.24) can alternatively be expressed in terms of the corresponding Dirac distributional stress momentum energy and background force density tensors, (d) $\hat{T}^{\mu \nu}$ and (d) $\hat{f}_{\mu}$, which are given for each (d-1)-brane in terms of the corresponding regular surface stress momentum energy and background force density tensors (d) $\bar{T}^{\mu \nu}$ and (d) $\bar{f}_{\mu}$ by

$$
{ }^{(\mathrm{d})} \hat{T}^{\mu \nu}=\|g\|^{-1 / 2} \int{ }^{(\mathrm{d})} \bar{d}^{(\mathrm{d})} \bar{T}^{\mu \nu} \delta^{\mathrm{n}}[x-x\{\sigma\}]
$$

and

$$
{ }^{(\mathrm{d})} \hat{f}_{\mu}=\|g\|^{-1 / 2} \int{ }^{(\mathrm{d})} \overline{\mathcal{S}}{ }^{(\mathrm{d})} \bar{f}_{\mu} \delta^{\mathrm{n}}[x-x\{\sigma\}] .
$$

The equivalent - more formally obvious but less explicitly meaningful - distributional versional version of the force balance law (2.24) takes the form

$$
\nabla_{\mu}\left(\sum_{\mathrm{d}}^{(\mathrm{d})} \hat{T}^{\mu \nu}\right)=\hat{f}_{\mu}
$$


where the total Dirac distributional force density is given in terms of the electromagnetic current distributions (2.16) and the vorticity flux distributions (2.18) by

$$
\hat{f}_{\mu}=F_{\rho \mu} \sum_{\mathrm{d}}^{(\mathrm{d})} \hat{J}^{\mu}+\frac{1}{2} N_{\rho \mu \nu} \sum_{\mathrm{d}}^{(\mathrm{d})} \hat{W}^{\mu \nu} .
$$

It is again left as an optional exercise for readers who are adept in the use of Dirac distributions to show that the system $(2.24),(2.25),(2.26)$ is obtainable from (2.29) and (2.30) by substituting (2.16), (2.18), (2.27), (2.28).

As an immediate corollary of (2.24), it is to be noted that for any vector field $k^{\mu}$ that generates a continuous symmetry of the background spacetime metric, i.e. for any solution of the Killing equations

$$
\nabla_{(\mu} k_{\nu)}=0
$$

one can construct a corresponding surface momentum or energy density current

$$
{ }^{(\mathrm{p})} \bar{P}^{\mu}={ }^{(\mathrm{p})} \bar{T}^{\mu \nu} k_{\nu},
$$

that will satisfy

$$
{ }^{(\mathrm{p})} \bar{\nabla}_{\mu}{ }^{(\mathrm{p})} \bar{P}^{\mu}=\sum_{\mathrm{d}=\mathrm{p}+1}{ }^{(\mathrm{d})} \lambda_{\mu}{ }^{(\mathrm{d})} \bar{P}^{\mu}+{ }^{(\mathrm{p})} \bar{f}_{\mu} k^{\mu} .
$$

In typical applications for which the n-dimensional background spacetime can be taken to be flat there will be $\mathrm{n}$ independent translation Killing vectors which alone (without recourse to the further $\mathrm{n}(\mathrm{n}-1) / 2$ rotation and boost Killing vectors of the Lorentz algebra) will provide a set of relations of the form (2.33) that together provide the same information as that in the full force balance equation (2.24) or (2.29).

Rather than the distributional version (2.29), it is the explicit version (2.24) of the force balance law that is directly useful for calculating the dynamic evolution of the brane support surfaces. Since the relation (2.29) involves $\mathrm{n}$ independent components whereas the support surface involved is only p-dimensional, there is a certain redundancy, which results from the fact that if the virtual displacement field $\xi^{\mu}$ is tangential to the surface in question it cannot affect the action. Thus if (p) $\perp_{\nu}^{\mu} \xi^{\nu}=0$, the condition (2.21) will be satisfied as a mere identity - provided of course that the field equations governing the internal fields of the system are satisfied. It follows that the non-redundent information governing the extrinsic motion of the p-dimensional support surface will be given just by the orthogonally projected part of (2.24). Integrating by parts, using the fact that, by (1.6) and (1.15), the surface gradient of the rank-(n-p) orthogonal projector ${ }^{(\mathrm{p})} \perp_{\nu}^{\mu}$ will be given in terms of the second fundamental tensor ${ }^{(\mathrm{p})} K_{\mu \nu}{ }^{\rho}$ of the p-surface by

$$
{ }^{(\mathrm{p})} \bar{\nabla}_{\mu}{ }^{(\mathrm{p})} \perp_{\rho}^{\nu}=-{ }^{(\mathrm{p})} K_{\mu \nu}{ }^{\rho}-{ }^{(\mathrm{p})} K_{\mu \nu}^{\rho},
$$


it can be seen that the extrinsic equations of motion obtained as the orthogonally projected part of (2.24) will finally be expressible by

$$
{ }^{(\mathrm{p})} \bar{T}^{\mu \nu(\mathrm{p})} K_{\mu \nu}{ }^{\rho}={ }^{(\mathrm{p})} \perp_{\mu}^{\rho}{ }^{(\mathrm{p})} f^{\mu} .
$$

It is to be emphasised that the formal validity of the formula that has just been derived is not confined to the variational models on which the above derivation is based, but also extends to dissipative models (involving effects such as external drag by the background medium 13] [14] 33] or mutual resistence between independent internal currents). The condition that even a nonconservative macroscopic model should be compatible with an underlying microscopic model of conservative type requires the existence (representing to averages of corresponding microscopic quantities) of appropriate stress momentum energy density and force density fields satisfying (2.35). This ubiquitously applicable formula is just the natural higher generalisation of "Newton's law" (equating the product of mass with acceleration to the applied force) in the case of a particle. The surface stress momentum energy tensor, ${ }^{(\mathrm{p})} \bar{T}^{\mu \nu}$, generalises the mass, and the second fundamental tensor, (p) $K_{\mu \nu}{ }^{\rho}$, generalises the acceleration. This can be seen from the fact that in the 1-dimensional case of a "pure" point particle (i.e. a monoplole) of mass $m$, the Lagrangian is given simply by ${ }^{(1)} \overline{\mathcal{L}}=-m$, so the 1-dimensional energy tensor is obtained in terms of the unit tangent vector $u^{\mu}\left(u^{\mu} u_{\mu}=-1\right)$ as ${ }^{(1)} \bar{T}^{\mu \nu}=m u^{\mu} u^{\nu}$. In this zero-brane case, the first fundamental tensor is simply given by ${ }^{(1)} \eta^{\mu \nu}=-u^{\mu} u^{\nu}$, and the second fundamental tensor is given in terms of the acceleration $\dot{u}^{\mu}=u^{\nu} \nabla_{\nu} u^{\mu}$ as ${ }^{(1)} K_{\mu \nu}{ }^{\rho}=u_{\mu} u_{\nu} \dot{u}^{\rho}$. Thus (2.35) can be seen to reduce in the case of a particle simply to the usual familiar form $m \dot{u}^{\rho}={ }^{(1)} \perp_{\mu}{ }_{\mu}^{(1)} f^{\mu}$.

\section{Perturbations and Curvature Effects beyond the Pure Brane Limit}

Two of the most useful formulae for the analysis of small perturbations of a string or higher brane worldsheet are the expressions for the infinitesimal Lagrangian (comoving) variation of the first and second fundamental tensors in terms of the corresponding comovings variation $d_{\mathrm{L}} g_{\mu \nu}$ of the metric (with respect to the comoving reference system). For the first fundamental tensor one easily obtains

$$
d_{\mathrm{L}} \eta^{\mu \nu}=-\eta^{\mu \rho} \eta^{\mu \sigma} d_{\mathrm{L}} g_{\rho \sigma}, \quad d_{\mathrm{L}} \eta^{\mu}{ }_{\nu}=\eta^{\mu \rho} \perp_{\nu}^{\sigma}{ }_{\nu} d_{\mathrm{L}} g_{\rho \sigma}
$$

and, by substituting this in the defining relation (1.10), the corresponding Lagrangian variation of the second fundamental tensor is obtained [35] as

$$
d_{\mathrm{L}} K_{\mu \nu}{ }^{\rho}=\perp_{\lambda}^{\rho} \eta_{\mu}^{\sigma} \eta_{\nu}^{\tau} d_{\mathrm{L}} \Gamma_{\sigma \tau}^{\lambda}+\left(2 \perp_{(\mu}^{\sigma} K_{\nu)}{ }^{\tau \rho}-K_{\mu \nu}{ }^{\sigma} \eta^{\tau \rho}\right) d_{\mathrm{L}} g_{\sigma \tau},
$$

where the Lagrangian variation of the connection (1.18) is given by the well known formula

$$
d_{\mathrm{L}} \Gamma_{\sigma \tau}^{\lambda}=g^{\lambda \rho}\left(\nabla_{(\sigma} d_{\mathrm{L}} g_{\tau) \rho}-\frac{1}{2} \nabla_{\rho} d_{\mathrm{L}} g_{\sigma \tau}\right)
$$


Since we are concerned here only with cases for which the background is fixed in advance so that the Eulerian variation $d_{\mathrm{E}}$ will vanish in (2.19), the Lagrangian variation of the metric will be given just by its Lie derivative with respect to the infinitesimal displacement vector field $\xi^{\mu}$ that generates the displacement of the worldsheet under consideration, i.e. we shall simply have

$$
d_{\mathrm{L}} g_{\sigma \tau}=2 \nabla_{(\sigma} \xi_{\tau)}
$$

It then follows from (3.3) that the Lagrangian variation of the connection will be given by

$$
d_{\mathrm{L}} \Gamma_{\sigma \tau}^{\lambda}=\nabla_{(\sigma} \nabla_{\tau)} \xi^{\lambda}-\mathcal{R}_{(\sigma \tau) \rho}^{\lambda} \xi^{\rho},
$$

where $\mathcal{R}_{\sigma \tau \rho}^{\lambda}$ is the background Riemann curvature (which will be negligible in typical applications for which the lengthscales characterising the geometric features of interest will be small compared with those characterising any background spacetime curvature). The Lagrangian variation of the first fundamental tensor is thus finally obtained in the form

$$
d_{\mathrm{L}} \eta^{\mu \nu}=-2 \eta_{\sigma}^{(\mu} \bar{\nabla}^{\nu)} \xi^{\sigma}
$$

while that of the second fundamental tensor is found to be given by

$$
\begin{array}{r}
d_{\mathrm{L}} K_{\mu \nu}{ }^{\rho}=\perp_{\lambda}^{\rho}\left(\bar{\nabla}_{(\mu} \bar{\nabla}_{\nu)} \xi^{\lambda}-\eta_{(\mu}^{\sigma} \eta_{\nu)}^{\tau} \mathcal{R}_{\sigma \tau \rho}^{\lambda} \xi^{\rho}-K_{(\mu \nu)}^{\sigma} \bar{\nabla}_{\sigma} \xi^{\lambda}\right)+ \\
\left(2 \perp_{(\mu}^{\sigma} K_{\nu) \tau}{ }^{\rho}-g_{\tau}^{\rho} K_{\mu \nu}{ }^{\sigma}\right)\left(\nabla_{\sigma} \xi^{\tau}+\bar{\nabla}^{\tau} \xi_{\sigma}\right) .
\end{array}
$$

It is instructive to apply the forgoing formulae to the case of a free pure brane worldsheet, meaning one for which there is no external force contribution so that the equation of extinsic motion reduces to the form

$$
\bar{T}^{\mu \nu} K_{\mu \nu}{ }^{\rho}=0 .
$$

On varying the relation (3.8) using (3.7) in conjunction with the orthogonality property (2.22) and the unperturbed equation (3.8) itself, the equation governing the propagation of the infinitesimal displacement vector is obtained in the form

$$
\perp_{\lambda}^{\rho} \bar{T}^{\mu \nu}\left(\bar{\nabla}_{\mu} \bar{\nabla}_{\nu} \xi^{\lambda}-\mathcal{R}_{\mu \nu \sigma}^{\lambda} \xi^{\sigma}\right)=-K_{\mu \nu}{ }^{\rho} d_{\mathrm{L}} \bar{T}^{\mu \nu} .
$$

The extrinsic perturbation equation (3.9) is by itself only part of the complete system of perturbation equations governing the evolution of the brane, the remaining equations of the system being those governing the evolution of whatever surface current 34 and other relevant internal fields on the supporting worldsheet may be relevant. The perturbations of such fields are involved in the source term on the right of (3.9), whose explicit evaluation depends on the specific form of the relevant currents or other internal fields. However it is not necessary to know the specific form of such internal fields for the purpose just of deriving the characteristic velocities of propagation of 
the extrinsic propagations represented by the displacement vector $\xi^{\mu}$, so long as they contribute to the source term on the right of the linearised perturbation equation (3.9) only at first differential order, so that the characteristic velocities will be completely determined by the first term on the left of (3.9) which will be the only second differential order contribution. It is apparent from (3.9) that under these conditions the equation for the characteristic tangent covector $\chi_{\mu}$ say will be given independently of any details of the surface currents or other internal fields simply [4] by

$$
\bar{T}^{\mu \nu} \chi_{\mu} \chi_{\nu}=0
$$

(It can be seen that the unperturbed surface stress momentum energy density tensor $\bar{T}^{\mu \nu}$ plays the same role here as that of the unperturbed metric tensor $g^{\mu \nu}$ in the analogous characteristic equation for the familiar case of a massless background spacetime field, as exemplified by electromagnetic or gravitational radiation.)

The linerised perturbation equation (3.9) will of course completely determine the evolution of the displacements by itself if there are no internal fields, i.e. in the case of the Dirac Gotu Nambu model determined by a surface Lagrangian of the trivial constant form

$$
\text { (d) } \overline{\mathcal{L}}=-m^{\mathrm{d}}
$$

where $m$ is a constant having the dimension of mass (which would be of the order of magnitude of the relevant Higgs mass scale in the case of a vacuum defect arising from the spontaneous symmetry breaking mechanism of the kind most commonly considered[5]) and $\mathrm{d}$ is the dimension of the worldsheet (i.e. $d=1$ for a simple point particle, $d=2$ for a string, and so on). In this rather degenerate special case the surface stress momentum energy density tensor determined according to the prescription (2.7) will be given simply by

$$
{ }^{(\mathrm{d})} \bar{T}^{\mu \nu}=-m^{\mathrm{d}(\mathrm{d})} \eta^{\mu \nu} .
$$

The unperturbed Dirac Gotu Nambu equation of motion is thus obtained in a form that is given independently of the dimension $d$ indicated by the prefix ${ }^{(d)}$ which may therefore be dropped - by the well known harmonicity condition that is expressible as the vanishing of the curvature vector,

$$
K^{\mu}=0 \text {. }
$$

The corresponding perturbation equation is obtained from (3.9) in the form

$$
\perp_{\lambda}^{\rho}\left(\bar{\nabla}^{\mu} \bar{\nabla}_{\mu} \xi^{\lambda}-\eta^{\mu \nu} \mathcal{R}_{\mu \nu \sigma}^{\lambda} \xi^{\sigma}\right)=2 K_{\mu \nu}{ }^{\rho} \bar{\nabla}^{\mu} \xi^{\nu} .
$$

As well as the compact tensorial version 35 given here, the litterature includes other equivalent but formally more complicated expressions [36] [37] (involving reference to internal coordinates or surface adapted frames of the kind discussed at the beginning of Section 1) that generalise earlier work restricted to the hypersurface supported ("wall" or membrane) special case [38] [39] 40] [41]. 
For most practical physical purposes the most useful generalisations of the Dirac-Goto-Nambu models governed by (3.12) and (3.13) are those of the very general category governed by (3.8) and (3.9) which allow for internal fields such as the currents that can be used, not only to represent the Witten type superconductivity effect in cosmic strings, but also also to represent the effect of ordinary elasticity in terrestrial applications, such as the strings of musical instruments which were already a subject of scientific investigation, albeit at an empirical rather than theoretical level, in the time of Pythagoras. However before proceeding to the discussion of such internal field effects in the following section, it is of interest to consider how the simple Dirac-Goto-Nambu model can be generalised in a way that goes beyond the "pure" brane description characterised in the free case by (3.8) and in the presence of an external force by (2.35). The distinguishing property of a "pure" brane model of the kind considered in the previous section is the condition that the action depends only on the undifferentiated background fields $g_{\mu \nu}, A_{\mu}, B_{\mu \nu}$, but not on their gradients. One of the most familiar kinds of example in which this condition fails to hold is that of an electromagnetically polarised medium, whose action 42 depends not just on the gauge field $A_{\mu}$ but also directly on the associated field $F_{\mu \nu}$ itself. However for lack of time and space we shall not consider such electromagnetic effects in the present section but will consider only the simplest category of "geodynamic" brane models, meaning those in which, as in the "pure" brane models of the Dirac-Goto-Nambu category, the action depends only on the imbedding geometry of the worldsheet and not on any other external internal fields. The simplest such extension of the "pure" Dirac-Goto-Nambu model (whose action is proportional just to the surface measure which depends only on $g_{\mu \nu}$ but not its derivatives) is based on a Lagrangian consisting not only of a constant term but also of terms proportional to the two independent scalars that can be constructed as quadratic functions of the first derivatives of the metric, namely $K_{\mu} K^{\mu}$ and $K_{\mu \nu \rho} K^{\mu \nu \rho}$. The inclusion of such "stiffness" terms has been suggested by Polyakov and others [43], one of the main reasons being allowance for the deviations from the "pure" Dirac-Goto-Nambu description of cosmological string [44 [45] [46] or domain wall 47 [48] [49] [5] defects that one would expect to arise if the curvature becomes too strong. The kind of Lagrangian constructed in this way, namely

$$
{ }^{(\mathrm{d})} \overline{\mathcal{L}}=-m^{\mathrm{d}}+\mathrm{b}^{\text {(d) }} K_{\rho^{(\mathrm{d})}} K^{\rho}-\mathrm{c}^{\text {(d) }} K_{\mu \nu \rho}{ }^{(\mathrm{d})} K^{\mu \nu \rho},
$$

where $m, \mathrm{~b}$, and $\mathrm{c}$ are constants, has recently been the subject of several investigations [52 [53] 54 555. 56. It is convenient to use the abbreviation

$$
\text { (d) } \Sigma^{\mu \nu \rho}=\mathrm{b}^{(\mathrm{d})} \eta^{\mu \nu(\mathrm{d})} K^{\rho}-\mathrm{c}^{(\mathrm{d})} K^{\mu \nu \rho}
$$

which enables the Lagrangian (3.15) to be expressed in condensed form as

$$
{ }^{(\mathrm{d})} \overline{\mathcal{L}}=-m^{\mathrm{d}}+{ }^{(\mathrm{d})} \sum_{\mu \nu \rho}{ }^{(\mathrm{d})} K^{\mu \nu \rho} .
$$

The variation needed for evaluating the change in such an "impure" brane action will thereby be 
obtainable from the formulae above in the corresponding form

$$
\|h\|^{-1 / 2} d_{\mathrm{L}}\left(\|h\|^{1 / 2} \overline{\mathcal{L}}\right)=d_{\mathrm{L}} \overline{\mathcal{L}}+\frac{1}{2} \overline{\mathcal{L}} \eta^{\mu \nu} d_{\mathrm{L}} g_{\mu \nu}
$$

(again dropping the explicit reference to the brane dimension d) with

$$
d_{\mathrm{L}} \overline{\mathcal{L}}=\left(\Sigma^{\lambda \rho \mu} K_{\lambda \rho}^{\nu}-2 \Sigma_{\rho}^{\lambda \mu} K_{\lambda}^{\nu \rho}\right) d_{\mathrm{L}} g_{\mu \nu}+\left(2 \Sigma^{\mu \lambda \nu}-\Sigma^{\mu \nu \lambda}\right) \nabla_{\lambda} d_{\mathrm{L}} g_{\mu \nu} .
$$

Although it is still possible to construct a formally symmetric stress momentum energy density tensor of the distributional type, the presence of the gradient term on the right of (3.19) will make it rather pathological, with not just a contribution proportional to a Dirac distribution as in the "pure" brane case described by (2.27) but also with a contribution proportional to the even more highly singular gradient of a Dirac distribution[55. In order to be able to continue working in terms of strictly regular surface supported field, it is necessary [56] to deal with the gradient dependence of the action in such an "impure" brane model by having recourse to the use of a total stress momentum energy tensor $\mathcal{T}^{\mu}{ }_{\nu}$ of the no longer no longer symmetric canonical type that can be read out from the variation formula

$$
\|h\|^{-1 / 2} d_{\mathrm{L}}\left(\|h\|^{1 / 2} \overline{\mathcal{L}}\right)=\mathcal{T}^{\mu}{ }_{\nu} \bar{\nabla}_{\mu} \xi^{\nu}+2 \Sigma_{\mu}{ }^{\rho \sigma} \mathcal{R}_{\rho \sigma}{ }^{\mu}{ }_{\nu} \xi^{\nu}-\bar{\nabla}_{\mu}\left(2 \Sigma_{\nu}{ }^{\mu \rho} \nabla_{\rho} \xi^{\nu}\right)
$$

that is obtained after substitution of (3.4) in (3.18) and (3.19). The regular but non-symmetric canonical surface stress momentum energy density tensor $\mathcal{T}^{\mu}{ }_{\nu}$ obtained in this way [56] is given by

$$
\mathcal{T}^{\mu}{ }_{\nu}=\widetilde{T}^{\mu}{ }_{\nu}+t^{\mu}{ }_{\nu}
$$

where $\widetilde{T}^{\mu \nu}$ is symmetric and purely tangential to the worldsheet (as a regular "geometric" stress momentum energy density tensor would be) with a merely algebraic dependence on the second fundamental tensor, being given by

$$
\widetilde{T}^{\mu}{ }_{\nu}=\widetilde{T}_{\nu}{ }^{\mu}=\mathcal{T}^{\mu}{ }_{\lambda} \eta^{\lambda}{ }_{\nu}=\overline{\mathcal{L}} \eta^{\mu}{ }_{\nu}-2 \Sigma_{\nu \rho}^{\lambda}{ }_{\nu}{ }_{\lambda}^{\mu \rho},
$$

while the remainder, which is of higher differential order, is expressible in terms of the third fundamental tensor (1.22) as

$$
t_{\nu}^{\mu}=\mathcal{T}_{\lambda}^{\mu} \perp_{\nu}^{\lambda}=2 \mathrm{c} \Xi_{\lambda \nu}^{\lambda \mu}-2 \mathrm{~b} \Xi_{\lambda \nu}^{\mu \lambda}
$$

which can conveniently be rewritten with the higher derivative contributions regrouped in the form

$$
t^{\mu}{ }_{\nu}=2(\mathrm{c}-\mathrm{b}) \Xi^{\mu}{ }_{\nu}+\mathrm{c} \eta^{\mu}{ }_{\rho} \eta^{\sigma \tau} \mathcal{R}^{\rho}{ }_{\sigma \tau \lambda} \perp_{\nu}^{\lambda},
$$

where

$$
\Xi_{\nu}^{\mu}=\Xi_{\lambda \nu}^{\mu \lambda}=\perp_{\nu}^{\lambda} \bar{\nabla}^{\mu} K_{\lambda}
$$


It is to be noticed that the total canonical surface stress momentum energy density tensor obtained in this way is is still automatically tangential to the worldshheet on its first (though no longer on its second) index, i.e.

$$
\perp_{\mu}^{\lambda} \mathcal{T}^{\mu}{ }_{\nu}=0,
$$

and that the higher derivative contribution proportional to the trace $\Xi^{\mu}{ }_{\nu}$ of the third fundamental tensor will drop out if the coefficients have the same value, $c=b$.

The application of the variation principle to the effect that the surface integral of the variation (3.20) should vanish for any displacement $\xi^{\mu}$ within a bounded neighbourhood can be seen to lead (via an application of Green's theorem as in the preceeding section) to dynamical equations 56 of the form

$$
\bar{\nabla}_{\mu} \mathcal{T}^{\mu}{ }_{\nu}=2 \Sigma_{\mu}{ }^{\rho \sigma} \mathcal{R}_{\rho \sigma \nu}{ }_{\nu}
$$

As in the pure Dirac-Gotu-Nambu case discussed above, the foregoing system of equations is partially redundant: although it involves $\mathrm{n}$ distinct spacetime vectorial equations, only $\mathrm{n}-\mathrm{d}$ of them are dynamically independent, namely those projected orthogonally to the d-dimensional worldsheet. The others are merely Noether identities which follow independently of the variation principle from the fact that a displacement $\xi^{\nu}$ that is purely tangential to the worldsheet merely maps it onto itself and thus cannot affect the action, as can be verified directly using the generalised Codazzi identity (1.23).

The lack of symmetry of $\mathcal{T}^{\mu}{ }_{\nu}$ means that the construction of the corresponding momentum current vector, $\mathcal{P}^{\mu}$ say, associated with a generic background spacetime Killing vector field $k^{\mu}$ will not be quite as simple as in the "pure" brane case for which an expression of the simple form (2.32) suffices. However using the well known fact that the Killing equation (2.31) entails the integrability condition

$$
\nabla_{\mu} \nabla_{\nu} k^{\rho}=\mathcal{R}_{\nu \rho \lambda}^{\rho} k^{\lambda}
$$

together with the observation that the antisymmetric part of the canonical stress momentum energy density tensor (3.22) is given according to (3.23) just by

$$
\mathcal{T}^{[\mu \nu]}=-2 \bar{\nabla}_{\lambda} \Sigma^{\lambda[\mu \nu]},
$$

it can be seen that, for any solution of (2.31), the ansatz 56]

$$
\mathcal{P}^{\mu}=\mathcal{T}^{\mu}{ }_{\nu} k^{\nu}+2 \Sigma^{\mu \nu}{ }_{\rho} \nabla_{\nu} k^{\rho}
$$

provides a surface current, $\mathcal{P}^{\mu}$, which satisfies the tangentiality condition

$$
\perp_{\mu}^{\lambda} \mathcal{P}^{\mu}=0
$$

and for which the strict surface conservation law,

$$
\bar{\nabla}_{\mu} \mathcal{P}^{\mu}=0,
$$


will hold whenever the equation of motion (3.25) is satisfied. It is to be remarked however that for a Killing vector of the irrotational kind for which $\nabla_{\mu} k_{\mu}$ vanishes altogether the second term (interpretable as a surface spin density contribution) in (3.30) will not contribute, i.e. an expression of the simpler form (2.32) will suffice. This applies in particular to the case of an ordinary translation generator in flat space, for which the corresponding conserved surface current will represent ordinary energy or linear momentum, whereas in the case of angular momentum the extra (spin density) term in (2.32) is indispensible.

The preceeding formulae all include allowance for arbitrary background curvature, but, to obtain the analogue of the non redundant version (3.8) of the equations of motion in a reasonably simple form, the restriction that the background spacetime be flat, i.e. $\mathcal{R}^{\mu}{ }_{\nu \rho \sigma}=0$, will now be imposed. This enables the required system of dynamical equations to be expressed 56 in the form

$$
\widetilde{T}^{\mu \nu} K_{\mu \nu \rho}=2(\mathrm{~b}-\mathrm{c}) \perp_{\rho}^{\nu} \bar{\nabla}_{\mu}\left(\perp_{\nu}^{\sigma} \bar{\nabla}^{\mu} K_{\sigma}\right)
$$

with the higher derivative terms grouped on the right hand side, which vanishes if $\mathrm{b}=\mathrm{c}$.

In the particular case 51] of a membrane, meaning a brane supported by a hypersurface of dimension $\mathrm{d}=\mathrm{n}-1$, the second fundamental tensor and its trace will be given in terms of the unit normal $\lambda_{\rho}$ (which in this case will be unique up to a choice of sign) by $K_{\mu \nu \rho}=K_{\mu \nu} \lambda_{\rho}$ and $K_{\rho}=K \lambda_{\rho}$ with $K=K_{\mu}^{\mu}$ where $K_{\mu \nu}$ is the second fundamental form (whose sign depends on that orientation chosen for the normal). In an ordinary 4-dimensional spacetime background, this membrane case corresponds to $\mathrm{d}=3$, for which the symmetric tangential part of the surface stress momentum energy density tensor will be therefore be obtainable from (3.22) in the form

$$
{ }^{(3)} \widetilde{T}^{\mu \nu}=-\left(m^{3}-\mathrm{b}^{(3)} K^{2}+\mathrm{c}^{(3)} K_{\sigma}^{\rho}{ }^{(3)} K_{\rho}^{\sigma}\right){ }^{(3)} \eta^{\mu \nu}-2 \mathrm{~b}^{(3)} K^{(3)} K^{\mu \nu}+2 \mathrm{c}^{(3)} K^{\lambda \mu}{ }^{(3)} K_{\lambda}{ }^{\nu} .
$$

In the case of a string with $\mathrm{d}=2$ one can use the fact that the trace free conformation tensor (1.20) will satisfy $2^{(2)} C^{\lambda}{ }_{\mu \rho}{ }^{(2)} C_{\lambda \nu}{ }^{\rho}={ }^{(2)} C_{\kappa \lambda \rho}{ }^{(2)} C^{\kappa \lambda \rho\left({ }^{(2)}\right.} \eta_{\mu \nu}$ to obtain a corresponding formula (which holds regardless of the background spacetime dimension $\mathrm{n}$ ) given 56 by

$$
{ }^{(2)} \widetilde{T}^{\mu \nu}=-m^{2(2)} \eta^{\mu \nu}+2(\mathrm{c}-\mathrm{b})^{(2)} C^{\mu \nu \rho(2)} K_{\rho} .
$$

\section{Conservative String Models as examples of perfect Branes}

It is reasonable to postulate that a "weak" energy condition of the kind formulated and justified by Hawking and Ellis 57 should hold for any pure p-brane model as a condition for physical realism as a macroscopic description of a $(\mathrm{p}+1)$-surface supported physical system at a classical level, meaning that the model's surface stress momentum energy density tensor (as introduced in section 2) should be such that the contraction $\bar{T}^{\mu \nu} \beta_{\mu} \beta_{\nu}$ is non negative for any vector $\beta^{\mu}$ that is timelike. Furthermore the causality condition to the effect that there should be no timelike 
characteristic covector (i.e. no superluminal propagation) can be seen from (3.10) to entail the further requirement (going marginally beyond the "weak" condition of Hawking and Ellis) that $\bar{T}^{\mu \nu} \beta_{\mu} \beta_{\nu}$ should be strictly positive if $\beta^{\mu}$ is timelike. This leads to the formulation of what may be called the "minimal" energy condition for a pure p-brane which is expressible as

$$
\beta^{\mu} \beta_{\mu}<0 \quad \Rightarrow \quad \bar{T}^{\mu \nu} \beta_{\mu} \beta_{\nu}>0 .
$$

A (pure) p-brane model will consequently be characterised by a well defined surface energy density, $U$ say, that is specifiable by an eigenvalue equation of the form

$$
\bar{T}^{\mu}{ }_{\nu} \beta^{\nu}=-U \beta^{\mu}
$$

where the corresponding eigenvector $\beta^{\mu}$ is distinguished by the requirement that it be tangential and non-spacelike:

$$
\perp_{\nu}^{\mu} \beta^{\nu}=0, \quad \beta^{\mu} \beta_{\mu} \leq 0 .
$$

It is apparent that the "minimal" energy condition (4.1) requires that the eigenvalue $U$ should be strictly positive unless $\beta^{\mu}$ is null in which case it may vanish:

$$
U \geq 0, \quad U=0 \quad \Rightarrow \quad \beta^{\mu} \beta_{\mu}=0
$$

In the Dirac-Goto-Nambu model that is most familiar to present day cosmologists, the eigenvector $\beta^{\mu}$ is indeterminate and the energy density $U$ is the same (in relativistic units such as are used here, with the speed of light set to unity) as the corresponding (surface) tension. However in general it is essential to distinguish the concept of energy density $U$ from the concept of the tension (as used in physics since the formulation of Hooke's law at the time of Newton) from which term "tensor" is derived. The (surface) tension scalar, for which we shall use the traditional symbol $T$, is defineable generically in a manner consistent with traditional usage, for a "pure" p-brane (i.e. a $(p+1)$-dimensionally supported system) of the kind considered here by decomposing the trace of the surface stress momentum energy density tensor in the form

$$
\bar{T}_{\mu}^{\mu}=-U-\mathrm{p} T .
$$

Appart from the degenerate Dirac-Goto-Nambu case for which $U$ and $T$ are actually equal, the simplest possibility is that of a perfect p-brane 1 , meaning one whose surface stress momentum energy density tensor is spacially (thus p-dimensionally) isotropic so that it is will be expressible, for a suitable choice of the normalisation of the eigenvector $\beta^{\mu}$ in (4.2) by

$$
\bar{T}^{\mu \nu}=\beta^{\mu} \beta^{\nu}-T \eta^{\mu \nu},
$$

where the required normalisation is given by

$$
U-T=\beta^{\mu} \beta_{\mu} \leq 0 .
$$


This category includes the case of the Dirac-Goto-Nambu model (3.12), which is obtained (with $T=m^{\mathrm{p}+1}$ ) by normalising the (in this case indeterminate) eigenvector to zero, i.e setting $\beta^{\mu}=0$. A more mundane example is provided by the familiar "improper" case $\mathrm{p}=\mathrm{n}-1$ (where $\mathrm{n}$ is the background dimension) of an ordinary (relativistic) perfect fluid with pressure $P=-T$. Although the tension $T$ is negative in the ordinary fluid case, it must be positive, as a condition for stability, for "proper" $\mathrm{p}$-branes of lower dimension, $\mathrm{p}<\mathrm{n}-1$. Whereas the membrane models (with $\mathrm{p}=2$ for $\mathrm{n}=4$ ) that are appropriate for the description of nautical sails will not in general have the isotropic form (4.6), a familiar everyday example of a membrane that will automatically be "perfect" in this sense is provided by the case of an ordinary soap bubble whose boundary hypersurface will be characterised by $0<T<<U$. The kind of application on which we shall concentrate in the following sections is that of a string, as given by $\mathrm{p}=1$, for which the "perfection" property (4.6) will always hold: isotropy cannot fail to apply in this 1-brane case because only a single space dimension is involved.

Before specialising to the (automatically perfect) string case, it is to be remarked that except when the $\beta^{\mu}$ is indeterminate (as in the Dirac-Goto-Nambu case), or null (as can occur in special subspaces where the current becomes null in a string model of the kind 31] 32] 34] appropriate for describing cosmic vortex defects of the "superconducting" kind proposed by Witten[7]) this eigenvector will determine a corresponding unit eigenvector,

$$
u^{\mu}=(U-T)^{-1 / 2} \beta^{\mu}, \quad u^{\mu} u_{\mu}=-1,
$$

specifying a naturally preferred rest frame, in terms of which (4.6) will be rewriteable in the standard form

$$
\bar{T}^{\mu \nu}=(U-T) u^{\mu} u^{\nu}-T \eta^{\mu \nu} .
$$

With respect to the frame so defined, it can be seen that the velocity $c_{\mathrm{E}}$ say of propagation of extrinsic perturbations (what are commonly referred to as "wiggles") of the perfect brane worldsheet will be given, according to the characteristic equation (3.10), by

$$
c_{\mathrm{E}}^{2}=\frac{T}{U} .
$$

By substituting (4.8) in the (3.3), and using the expression (1.16) for the acceleration $\dot{u}^{\mu}$ of the preferred unit vector (4.8), the extrisic equation governing the free motion of the support surface of any (proper) perfect brane is reducible to the standard form [4] [34]

$$
c_{\mathrm{E}}^{2} K^{\mu}=\left(1-c_{\mathrm{E}}^{2}\right) \perp_{\nu}^{\mu} \dot{u}^{\nu} .
$$

In the Dirac-Goto-Nambu case one has $U=T$ and hence $c_{\mathrm{E}}^{2}=1$, (i.e. the "wiggle" speed is that of light) so that the right hand side of (4.11) will drop out, leaving the harmonicity condition that is expressed by the vanishing of the curvature vector given by (1.19). At the opposite extreme from 
this relativistic limit case, one has applications to such mundane examples as that of an ordinary soap bubble membrane or violin string for which one has $c_{\mathrm{E}}^{2}<<1$.

Except in the Dirac-Goto-Nambu limit case, the extrinsic equation of motion (4.12) will need to be supplemented by the dynamic equations governing the evolution of the internal fields, and in particular of $T, U$, and $u^{\mu}$, on the brane surface. The simplest non trivial possibility is the case of a perfect brane model that is "barotropic", meaning that its tension $T$ (or equivalently, in the case of a fluid, its pressure $P$ ) is a function only of the energy density $U$ in the preferred rest frame. In this barotropic or "perfectly elastic" case, the complete set of dynamical equations governing the internal evolution of the perfect brane will be provided just by the tangential projection of the force balance law (2.24) which, in the free case to which the discussion of the present section is restricted, has the simple form

$$
\eta_{\nu}^{\lambda} \bar{\nabla}_{\mu} \bar{T}^{\mu \nu}=0 .
$$

In the barotropic case (which includes the kind of cosmic string models [31] 32] [34] appropriate for describing the Witten type superconducting vaccuum vortices [7] whose investigation provided the original motivation for developing the kind of analysis presented here) experience of the "improper" case of an (n-1)-brane, i.e. the familiar example of an ordinary barotropic perfect fluid, suggests the convenience of introducing an idealised particle number density, $\nu$ say, and a corresponding (relativistic) chemical potential, or effective mass per idealised particle, $\mu$ say, that are specified modulo an arbitrary normalisation factor by the equation of state according to a prescription of the form

$$
\ln \nu=\int \frac{d U}{U-T}, \quad \ln \mu=\int \frac{d T}{T-U},
$$

which fixes them modulo a pair of constants of integration which are not allowed to remain independent but are related by the imposition of the restraint condition

$$
\mu \nu=U-T .
$$

The quantities introduced in this way can be used for defining a surface particle current density vector $\nu^{\rho}$ and a corresponding dynamically conjugate energy - momentum (per particle) covector $\mu_{\rho}$ that are given by

$$
\nu^{\rho}=\nu u^{\rho}, \quad \mu_{\rho}=\mu u_{\rho},
$$

in terms of which the generic expression (4.9) of the surface stress momentum energy density tensor of a perfect brane can be rewritten in the form

$$
\bar{T}_{\sigma}^{\rho}=\nu^{\rho} \mu_{\sigma}-T \eta^{\rho}{ }_{\sigma},
$$

whose divergence can be seen to be given identically by

$$
\bar{\nabla}_{\rho} \bar{T}_{\sigma}^{\rho}=\mu_{\sigma} \bar{\nabla}_{\rho} \nu^{\rho}+2 \nu^{\rho} \bar{\nabla}_{[\rho} \mu_{\sigma]}-T K_{\sigma} .
$$


In the free case for which this divergence vanishes, projection orthogonal to the worldsheet gives back the extrinsic dynamical equation (4.11), while by contraction with $\nu^{\rho}$ one obtains the simple surface current conservation law

$$
\bar{\nabla}_{\rho} \nu^{\rho}=0
$$

The remainder of the system of equations of motion of the perfect brane can hence be obtained from (4.12), as a surface generalised version of the standard perfect fluid momentum transport law 58$]$, in the form

$$
\eta_{\nu}^{\rho} u^{\mu} \bar{\nabla}_{[\mu} \mu_{\rho]}=0
$$

which is the integrability condition for the existence of a surface potential function, $\varphi$ say, such that $\mu_{\rho}=\bar{\nabla}_{\rho} \varphi$.

Appart from the extrinsic perturbations of the world sheet location itself, which propagate with the "wiggle" speed $c_{\mathrm{E}}$ (relative to the frame deterined by $u^{\mu}$ ) as already discused, the only other kind of perturbation mode that can occur in a barytropic string are longitudinal modes specified by the variation of $U$ or equivalently of $T$ within the world sheet. As in the "improper" special case of an ordinary perfect fluid, for which they are interpretable just as ordinary sound waves, such longitudinal "woggle" perturbations can easily be seen from (4.16) and (4.17) to have a relative propagation velocity $c_{\mathrm{L}}$ say that is given 34 by

$$
c_{\mathrm{L}}^{2}=\frac{\nu d \mu}{\mu d \nu}=-\frac{d T}{d U}
$$

In order for a barotropic model to be well behaved, $c_{\mathrm{E}}^{2}$ and $c_{\mathrm{L}}^{2}$ must of course both be positive, in order for the velocities to be real (as a condition for local stability), and they must also both be less than unity in order to avoid superluminal propagation (as a condition for local causality). An important qualitative question that arises for any proper barotropic p-brane, i.e. one with $1 \leq \mathrm{p}<\mathrm{n}-$ 1 (excluding the trivial case $\mathrm{p}=0$ of a point particle, and at the other extreme the "improper" case $\mathrm{p}=\mathrm{n}-1$ of a fluid) is whether the extrinsic propagation speed is subsonic, meaning that "wiggles" go slower than "woggles", i.e $c_{\mathrm{E}}<c_{\mathrm{L}}$, transonic, meaning that the speeds are the same, $c_{\mathrm{E}}=c_{\mathrm{L}}$ or supersonic, meaning that "wiggles" go faster than "woggles", $c_{\mathrm{E}}>c_{\mathrm{L}}$. Much of the earliest work 59 60] 61] 62] 63] 65] on the Witten type superconducting vaccuum vortex phenomena was implicitly based on use of a string model of subsonic type, but a more careful analysis of the internal structure of the Witten vortex by Peter 66 67 60 60 has since shown that models of supersonic type are more appropriate. On the other hand such everyday examples as that of an ordinary violin string are subsonic, a condition that is sufficient 69 though not necessary 70 for stability of the corresponding circular centrifugally supported loop configurations 701 [72] [73 that will be discussed later on.

A particularly important special case is that of a barytropic p-brane of permanently transonic type, meaning one characterised by an equation of state of the non dispersive constant product 
form

$$
U T=m^{2 \mathrm{p}+2},
$$

where $m$ is a constant having the dimensions of a mass, for which application of the formulae (4.10) and (4.18) gives $c_{\mathrm{E}}^{2}=c_{\mathrm{L}}^{2}$ not just for some critical transition value of $U$ or $T$ (as can occur for other kinds of equation of state) but for all values. In the string case, $p=1$, it can easily be demonstrated [4 that this non dispersive permanently transonic model is represents the outcome of a (rather artificial) dimensional reduction process first suggested by Nielsen 75$]$ [76] [77] [78]. As will be described below, this model can be shown 79 to be governed by equations of motion that are explicitly integrable in a flat empty background, and it can be used [79] [80] to provide what (contrary to a misleading claim 81 that has been made) can be a highly accurate description of the macroscopic effect of "wiggles" in an underlying cosmic string of the simple Goto-Nambu type.

\section{$5 \quad$ Essentials of Elastic String Dynamics}

Between the hypersurface supported case of a membrane and the curve supported case of a point particle, the only intermediate kind of brane that can exist in 4-dimensions is that of 1-brane, i.e. a string model, which (for any background dimension $n$ ) will have a first fundamental tensor that is expressible as the square of the antisymmetric unit surface element tensor $\mathcal{E}^{\mu \nu}=-\mathcal{E}^{\nu \mu}$ given by (1.28):

$$
\eta^{\mu}{ }_{\nu}=\mathcal{E}^{\mu}{ }_{\rho} \mathcal{E}_{\nu}^{\rho}
$$

In the case of string (to which the remainder of this article will be devoted) the symmetric surface stress momentum energy tensor $\bar{T}^{\mu \nu}$ that is well defined for any "pure" (i.e. unpolarised) model will not only be expressible in the generic form (4.6) that expresses the (in the string case trivial) property of spacial anisotropy with respect to the preferred timelike or null eigenvector $\beta^{\mu}$, but it will also be expressible generically in bicharacteristic form as

$$
\bar{T}^{\mu \nu}=\beta_{+}^{[\mu} \beta_{-}^{\nu]},
$$

in terms of a pair of timelike or null tangent vectors $\beta_{+}^{\mu}$ that can be seen to be extrinsic bicharacteric vectors, meaning that they lie respectively allong the two ("right moving" and "left moving") directions of propagation of extrinsic perturbations, and are thus respectively orthogonal to the covectors $\chi_{\mu}$ given by the extrinsic characteristic equation (3.10) whose solutions can be seen from (5.2) to be given by $\beta_{+}^{\mu} \chi_{\mu}=0$ or $\beta_{-}^{\mu} \chi_{\mu}=0$. The expression (5.2) does not completely determine the bicharacteristic vectors $\beta_{ \pm}^{\mu}$, but leaves open the possibility of a reciprocal multiplicative rescaling of their magnitudes, subject to the condition that their scalar product remains invariant, its value being obtainable by comparison of (5.2) with (4.6) as

$$
\beta_{+\mu} \beta_{-}^{\mu}=\bar{T}_{\nu}^{\nu}=-(U+T) .
$$


The geometric mean of the magnitudes of the bicharacteristic vectors remains similarly invariant, with value given by the magnitude of the preferred eigenvector $\beta^{\mu}$ as given by (4.7), i.e.

$$
\left(\beta_{+}{ } \beta_{+}{ }^{\mu}\right)\left(\beta_{-\nu} \beta_{-}^{\nu}\right)=\left(\beta_{\mu} \beta^{\mu}\right)^{2}=(U-T)^{2}
$$

To obtain the equations of motion of the string we need to use the force balance equation (2.25), which just takes the form

$$
\bar{\nabla}_{\mu} \bar{T}^{\mu \nu}=\bar{f}^{\nu}
$$

for a string that is isolated, where $\bar{f}^{\mu}$ (which simply vanishes in the free case) is the force exerted by any background fields, such as the electromagnetic field and ambient (Higgs or other) fluid that are allowed for in the explicit expression (2.26). (The only difference if the string were not isolated, i.e. if it belonged to the boundary of one or more attached membranes, is that an additional contact force contribution $\check{f}^{\mu}$ of the form (2.25) would also be needed as well as the background contribution $\bar{f}^{\mu}$.) Using the Weingarten integrability condition (1.13) which is equivalent in the string case to the the projected Lie commutativity condition

$$
\perp_{\nu}^{\rho}\left(\beta_{+}{ }^{\mu} \bar{\nabla}_{\mu} \beta_{-}^{\nu}-\beta_{-}{ }^{\mu} \bar{\nabla}_{\mu} \beta_{+}{ }^{\nu}\right)=0
$$

it can be seen from (5.2) that the extrinsic equations of motion governing the evolution of the string worldsheet will therefore 34 be expressible in characteristic form as

$$
\perp_{\nu}^{\rho} \beta_{ \pm}^{\mu} \bar{\nabla}_{\mu} \beta_{\mp}^{\nu}=\perp_{\mu}^{\rho} \bar{f}^{\mu},
$$

for either choice of the sign \pm , one version being obtainable from the other and vice versa by (5.6).

Except where one of the bicharacteristic vectors $\beta_{ \pm}{ }^{\mu}$ is null, which by (5.5) will only occur where $U=T$ (as is everywhere the case for the Goto-Nambu model, for which both bicharacteristic vectors are null everywhere) it will be possible to fix their normalisation by requiring that they have the same magnitude, $\beta_{ \pm}{ }_{\mu} \beta_{ \pm}^{\mu}=-(T-U)$ and hence to define a corresponding pair of unit bicharacteristic vectors

$$
u_{ \pm}^{\mu}=(U-T)^{-1 / 2} \beta_{ \pm}^{\mu}, \quad u_{ \pm}^{\mu} u_{ \pm}^{\mu}=-1
$$

in terms of which the unit timelike eigenvector $u^{\mu}$ introduced in (4.8), together with an orthogonal spacelike unit eigenvalue $v^{\mu}$, will be given by

$$
u^{\mu}=\frac{\sqrt{1-c_{\mathrm{E}}^{2}}}{2}\left(u_{+}{ }^{\mu}+u_{-}{ }^{\mu}\right), \quad v^{\mu}=\frac{\sqrt{1-c_{\mathrm{E}}{ }^{2}}}{2 c_{\mathrm{E}}}\left(u_{+}{ }^{\mu}-u_{-}{ }^{\mu}\right) .
$$

Such mutually orthogonal unit eigenvectors constitute a preferred diad, in terms of which the antisymmetric unit surface element $\mathcal{E}^{\mu \nu}$ and the (first) fundamental tensor $\eta^{\mu \nu}$ of the string worldsheet will be given by

$$
\mathcal{E}^{\mu \nu}=u^{\mu} v^{\nu}-v^{\mu} u^{\nu}, \quad \eta^{\mu \nu}=-u^{\mu} u^{\nu}+v^{\mu} v^{\nu},
$$


and in terms of which the standard form (4.9) for the surface stress momentum energy density tensor will reduce to the simple form

$$
\bar{T}^{\mu \nu}=U u^{\mu} u^{\nu}-T v^{\mu} v^{\nu} .
$$

Proceeding directly from this last form, the extrinsic dynamical equation (5.6), i.e. the surface orthogonal projection of (5.5), can be rewritten as

$$
\perp_{\nu}^{\mu}\left(U \dot{u}^{\nu}-T v^{\prime \nu}\right)=\perp_{\nu}^{\mu} \bar{f}^{\nu},
$$

using the notation

$$
\dot{u}^{\mu}=u^{\nu} \nabla_{\nu} u^{\nu}, \quad v^{\mu}=v^{\nu} \nabla_{\nu} v^{\nu} .
$$

in terms of which the worldsheet curvature vector is expressible as

$$
K^{\mu}=\perp_{\nu}^{\mu}\left(v^{\prime \nu}-\dot{u}^{\nu}\right) .
$$

These last formulae illustrate a special feature distinguishing string models from point particle models on one hand and from higher dimensional brane models on the other, namely the dual symmetry 31] that exists at a formal level between the timelike eigenvector $u^{\mu}$ and the associated eigenvalue $U$ on one hand, and the the dynamically conjugate quantities that are the spacelike eigenvector and $v^{\mu}$ and the associated eigenvalue $T$. This feature is particularly informative in the barotropic or "perfectly elastic" case discussed at the end of the previous section, i.e. in the case when the internal dynamics is controlled just by an equation of state giving the energy density $U$ as a function of the tension $T$ only, since in there will be a corresponding duality relation between the number density $\nu$ and the associated chemical potential $\mu$ as introduced in (4.13), which is equivalent to the mutually dual pair of differential defining relations

$$
d U=\mu d \nu, \quad d T=-\nu d \mu .
$$

Defining the surface duals of the current vector $\nu^{\rho}$ and momentum covector $\mu_{\rho}$ given by (4.15) as

$$
\star \nu_{\rho}=\mathcal{E}_{\rho \sigma} \nu^{\sigma}=\nu v_{\rho}, \quad \star \mu^{\rho}=\mathcal{E}^{\rho \sigma} \mu_{\sigma}=\mu v^{\rho},
$$

one can use (4.17) to obtain the force balance equation for a barotropic string model in the self dual form

$$
\mu_{\rho} \bar{\nabla}_{\sigma} \nu^{\sigma}+\star \nu_{\rho} \nabla_{\sigma} \star \mu^{\sigma}+\perp_{\rho}^{\sigma}\left(U \dot{u}^{\sigma}-T v^{\prime \sigma}\right)=\bar{f}_{\rho},
$$

whose orthogonally projected part gives back the self dual extrinsic force balance equation (5.13), while its tangentially projected part gives a mutually dual pair of surface current source equations of the form

$$
(U-T) \bar{\nabla}_{\sigma} \nu^{\sigma}=-\nu^{\rho} \bar{f}_{\rho}, \quad(U-T) \bar{\nabla}_{\sigma} \star \mu^{\sigma}=\star \mu^{\rho} \bar{f}_{\rho} .
$$


It can be seen from this that, like the surface number current density $\nu^{\sigma}$, the dual current $\star \mu^{\sigma}$ will also be conserved in a free barotropic string, i.e. when the external force $\bar{f}_{\rho}$ is absent.

It is useful 31 to introduce a dimensionless state function called the "characteristic potential" $\vartheta$ say that is constructed in such a way as to be self dual according to the differential relation

$$
d \vartheta=\sqrt{\frac{d \mu d \nu}{\mu \nu}} .
$$

This function is convenient for the purpose of writing the internal equations of motion obtained by tangential projection of (5.5) in a characteristic form that will be the analogue of the characteristic version (5.7) of the extrinsic equations of motion obtained by the orthogonal projection of (5.5). The longitudinal bicharacteristic unit vectors $\ell_{ \pm}{ }^{\mu}$ say (the analogues for the internal sound type waves of the extrinsic bicharacteristic unit vectors ${u_{ \pm}}^{\mu}$ introduced above) are defineable by

$$
\ell_{ \pm}^{\mu}=\left(1-c_{\mathrm{L}}{ }^{2}\right)^{-1 / 2}\left(u^{\mu} \pm c_{\mathrm{L}} v^{\mu}\right), \quad \ell_{ \pm}{ }^{\mu} \ell_{ \pm}^{\mu}=-1
$$

where $c_{\mathrm{L}}$ is the longitudinal characteristic speed as given by (4.20). In terms of these, the tangentially projected part of the force balance equation (5.5) is convertible [31 to the form of a pair of scalar equations having the characteristic form

$$
(U-T) \ell_{ \pm}^{\rho}\left(\bar{\nabla}_{\rho} \vartheta \mp v_{\nu} \bar{\nabla}_{\rho} u^{\nu}\right)= \pm \mathcal{E}_{\mu \nu} \ell_{\mp}^{\mu} \bar{f}^{\nu}
$$

from which it is directly apparent that, as asserted in the previous section, the state function $c_{\mathrm{L}}$, as given by (4.20), is indeed the characteristic speed of longitudinal "woggle" propagation relative to the preferred frame specified by the timelike eigenvector $u^{\mu}$. When rewritten in terms of the unit bicharacteristic vectors $u_{ \pm}{ }^{\mu}$ that are the extrinsic analogues of $\ell_{ \pm}{ }^{\mu}$, the corresponding extrinsic equations (5.7) take the form

$$
(U-T) \perp_{\nu}^{\mu} u_{ \pm}^{\rho} \bar{\nabla}_{\rho} u_{\mp}^{\nu}=\perp_{\nu}^{\mu} \bar{f}^{\nu},
$$

(It is to be noted that the corresponding equation (32) at the end of the original discussion 31] of the characteristic forms of the equations of string motion contains a transcription error whereby a proportionality factor that should have been $\sqrt{(U-T) T}$ was replaced by its non-relativistic - i.e. low tension - limit, namely $\sqrt{U T}$.)

What essentially distinguishes different physical kinds of "perfectly elastic", i.e. barotropic, string model from one another is the form of the equation of state, which depends on the microsropic internal details of the vacuum defect (or other structure) that the string model is supposed to represent. In a cosmological context, the most important special case, which will be discussed in detail later on, is given by the constant product formula (4.21), but for terrestrial applications the most useful kind of equation of state for general purposes is the one named after Robert Hooke, who 
is recogniseably the principal founder of string theory as a branch of physics in the modern sense of the word. (As for superstring theory, it remains to be seen whether it will become established as a branch of physics or just as a topic of mathematics.) Hooke's famous law to the effect that the tension, $T$ is proportional to the extension, i.e. the change in $1 / \nu$ in the notation used here, provides a very good approximation in the low tension limit that is exemplified by applications such as that of an ordinary violin string. This law is expressible within the present scheme as

$$
U(Y+T)=U_{0} Y+\frac{1}{2} T^{2},
$$

where $U_{0}$ is a constant interpretable as the rest frame energy in the fully relaxed (zero tension) limit, and $Y$ is another constant interpretable as what is known (after another pioneer worker on the subject) as Young's modulus.

A more general category of non-barotropic string models is of course required, not only in such terrestrial applications as (above ground) electric power transmission cables (the subject of my first research project as an undergraduate working working for commercial industry during a vacation), but also for many cosmologically relevant cases that might be envisaged (such as that of a "warm superconducting string" [82]) in which several independent currents are present. Nevertheless most of the cosmological string dynamical studies that have been carried out so far have been restricted (not just for simplicity, but also as a very good approximation in wide range of circumstances) to models of the the "perfectly elastic" barotropic type, and more specifically to the category (including the Hookean and Goto Nambu examples as opposite extreme special cases) of string models describable by a Lagrangian of the form

$$
\overline{\mathcal{L}}=\Lambda+\bar{J}^{\mu} A_{\mu}+\bar{W}^{\mu \nu} B_{\mu \nu}
$$

in which, in order to satisfy the vorticity flux conservation requirement (as derived in Section 2 from the condition of invariance with respect to gauge changes in the background Kalb-Ramond field $B_{\mu \nu}$ ) one must have

$$
\bar{W}^{\mu \nu}=\kappa \mathcal{E}^{\mu \nu},
$$

for some fixed (quantised) circulation value $\kappa$ say, while another fixed coupling constant, $e$ say, specifies the electromagnetic current density, in the form

$$
\bar{J}^{\mu}=e c^{\mu},
$$

in terms of the only independent field variable on the world sheet, which can be taken to be a stream function $\psi$ which enters only via the corresponding identically conserved number density current

$$
c^{\mu}=\mathcal{E}^{\mu \nu} p_{\nu}, \quad p_{\nu}=\bar{\nabla}_{\nu} \psi,
$$

whose squared magnitude,

$$
\chi=c_{\mu} c^{\mu}=-p^{\mu} p_{\nu}=-h^{i j} \psi_{i} \psi_{j},
$$


is the only argument in a (generically non-linear) master function,

$$
\Lambda=\Lambda\{\chi\}
$$

Previous studies of cosmic strings have mostly been restricted to cases involving allowance for a Kalb-Ramond coupling (which is relevant for vortex defects of "global type" [27] 83] 84] in the absence of an internal current, or alternatively to cases (such as are exemplified]31]32 by Witten's local superconducting string mechanism[7]) involving allowance for an internal current in the absence of Kalb-Ramond coupling. However there is no particular difficulty in enviseaging the presence of both kinds of coupling simultaneously as is done here. A new result that is obtained thereby (in Section 6) is that for stationary and other symmetric configurations, the effect of the coupling to tht Kalb Ramond background is expressible as that of a fictitious extra electromagnetic field contribution.

It is the specific form of the master function that determines the relevant equation of state or if its range of validity is sufficiently extensive, the pair of equations of state, corresponding to the qualitatively different regimes distinguished by the positivity or negativity of $\chi$ - by which the dynamics of the model is governed. The derivative of the master function provides a quantity

$$
\mathcal{K}=2 \frac{d \Lambda}{d \chi}
$$

in terms of which the surface stress momentum energy density tensor defined by (2.7) will be given by

$$
\bar{T}^{\mu \nu}=\mathcal{K} p^{\mu} p^{\nu}+\Lambda \eta^{\mu \nu}
$$

This only depends on the internal field gradient and on the imbedding and the background geometry: it involves neither the background electromagnetic and Kalb Ramond fields nor their respective coupling constants $e$ and $\kappa$. The role of the latter is just to determine the background force density, which will be given, according to the general formula (2.26), by

$$
\bar{f}_{\rho}=e F_{\rho \mu} c^{\mu}+\frac{\kappa}{2} N_{\rho \mu \nu} \mathcal{E}^{\mu \nu} .
$$

Unlike the force density, the stress momentum energy density tensor, as given by (5.32), is formally invariant under a duality transformation $\llbracket$ 还 31 whereby the variational momentum covector $p_{\mu}$, the current $c^{\mu}$, and the master function, $\Lambda$ itself, are interchanged with their duals, as denoted by a tilde, which are definable by

$$
\tilde{c}^{\mu}=\mathcal{K} p^{\mu}, \quad \tilde{p}_{\mu}=\mathcal{K} c_{\mu}, \quad \tilde{\chi}=\tilde{c}_{\mu} \tilde{c}^{\mu}=-\tilde{p}^{\nu} \tilde{p}_{\nu},
$$

and by the relation

$$
\chi \mathcal{K}=\Lambda-\tilde{\Lambda}=-\tilde{\chi} \tilde{\mathcal{K}},
$$


with

$$
\tilde{\mathcal{K}}=2 \frac{d \tilde{\Lambda}}{d \tilde{\chi}}=\mathcal{K}^{-1}, \quad \tilde{\chi}=-\mathcal{K}^{2} \chi
$$

In terms of these quantities the stress momentum energy density tensor can be rewritten in either of the equivalent mutually dual canonical forms

$$
\tilde{c}^{\mu} p_{\nu}+\Lambda \eta^{\mu}{ }_{\nu}=\bar{T}_{\nu}^{\mu}=c^{\mu} \tilde{p}_{\nu}+\tilde{\Lambda} \eta^{\mu}{ }_{\nu}
$$

Substitution of these respective expressions in (5.5) then gives the force balance equation in the equivalent mutually dual forms

$$
p_{\rho} \bar{\nabla}_{\mu} \tilde{c}^{\mu}+2 \tilde{c}^{\mu} \bar{\nabla}_{[\mu} p_{\rho]}+\Lambda K_{\rho}=\bar{f}_{\rho}=\tilde{p}_{\rho} \bar{\nabla}_{\mu} c^{\mu}+2 c^{\mu} \bar{\nabla}_{[\mu} \tilde{p}_{\rho]}+\tilde{\Lambda} K_{\rho} .
$$

The independent internal equations of motion of the system are obtainable by contraction with the independent mutually orthogonal tangent vectors $p^{\rho}$ and $\tilde{p}^{\rho}$, the latter giving what is just a kinematic identity, since, by construction according to the prescription given above, one automatically has

$$
\bar{\nabla}_{\mu} c^{\mu}=0=\mathcal{E}^{\rho \mu} \nabla_{\mu} p_{\rho}, \quad \tilde{p}^{\rho} f_{\rho}=0 .
$$

The only part of the internal equations of motion that is properly dynamical (from the point of view of the variation principle with respect to $\Lambda$ ) is thus just the part got by contraction with $p^{\rho}$, which gives

$$
-\chi \bar{\nabla}_{\mu} \tilde{c}^{\mu}=p^{\rho} \bar{f}_{\rho}=\chi \mathcal{E}^{\rho \mu} \bar{\nabla}_{[\mu} \tilde{p}_{\rho]}, \quad \quad p^{\rho} \bar{f}_{\rho}=e \chi \mathcal{E}^{\mu \rho} F_{\mu \rho} .
$$

It is to be noted that unlike the electromagnetic force, the Joukowski force (interpretable as a manifestation of the Magnus effect) arising from the Kalb-Ramond coupling in (5.33) will always act orthogonally to the world sheet and thus will not affect the internal dynamics of the string. It is evident that the internal force balance equation (5.40) can be rewritten simply as

$$
\mathcal{E}^{\mu \rho}\left(2 \nabla_{[\mu} \tilde{p}_{\rho]}+e F_{\mu \rho}\right)=0
$$

which can be construed as the (Poincaré type) integrability condition for the local existence of a scalar $\varphi$ on the worldsheet in terms of which the dual momentum will simply be given as the tangentially projected gauge covariant derivative:

$$
\tilde{p}_{\rho}=\bar{D}_{\rho} \varphi, \quad \bar{D}_{\rho}=\eta_{\rho}^{\mu} D_{\mu}, \quad D_{\mu}=\nabla_{\mu}-e A_{\mu} .
$$

In applications to Witten type superconducting strings, this field $\varphi$ will be interpretable 31 as being proportional to - and therefore with respect to suitably scaled units equal to - the phase of an underlying complex bosonic field. The scalar $\varphi$ can be used as the primary independent variable, instead of $\psi$, in an alternative (dynamically conjugate) variational formulation using a Lagrangian constructed from $\tilde{\Lambda}$ instead of $\Lambda$. 
If its range of definition is sufficiently extended, the master function $\Lambda$ will determine not just one but a pair of distinct equations of state, one applying to the "magnetic" regime where the current $c^{\mu}$ is spacelike so that $\chi$ is positive, and the other applying to the "electric" regime where the current $c^{\mu}$ is timelike so that the $\chi$ is negative. In either of these distinct regimes - though not on the critical intermediate "null state" locus where the current is lightlike so that $\chi$ vanishes - the mutually dual canonical forms (5.37) of the stress momentum energy density tensor are replaceable by the equivalent more manifestly symmetric standard form

$$
\bar{T}^{\mu \nu}=\frac{\Lambda}{\chi} c^{\mu} c^{\nu}+\frac{\tilde{\Lambda}}{\tilde{\chi}} \tilde{c}^{\mu} \tilde{c}^{\nu} .
$$

By comparison with the corresponding expression (5.11) it can thus be seen that the physical interpretation - as energy density $U$, tension $T$, particle number density $\nu$ and effective mass per particle $\mu$ - of the state functions introduced in the variational function theat has just been given will be given, in the "magnetic" regime where $c^{\mu}$ is spacelike (the first possibility to be considered in the early work on the Witten mechanism[7]), by

$$
\Lambda=-T, \quad \tilde{\Lambda}=-U, \quad \chi=\mu^{2}, \quad \tilde{\chi}=-\nu^{2}, \quad \mathcal{K}=\frac{\nu}{\mu},
$$

with

$$
c^{\rho}=\mu v^{\rho}, \quad \tilde{c}^{\rho}=\nu u^{\rho}, \quad p_{\rho}=\mu u_{\rho}, \quad \tilde{p}_{\rho}=\nu v_{\rho} .
$$

On the other hand in the "electric" regime where $c^{\mu}$ is spacelike, one obtains what in general will be a different equation of state given parametrically by the dual set of relations

$$
\Lambda=-U, \quad \tilde{\Lambda}=-T \quad \chi=-\nu^{2}, \quad \tilde{\chi}=\mu^{2}, \quad \mathcal{K}=\frac{\mu}{\nu},
$$

with

$$
c^{\rho}=\nu u^{\rho}, \quad \tilde{c}^{\rho}=\mu v^{\rho}, \quad p_{\rho}=\nu v_{\rho}, \quad \tilde{p}_{\rho}=\mu u_{\rho} .
$$

In realistic conducting string models one can not expect the appropriate master function $\Lambda\{\chi\}$ to be given exactly by a simple analytic formula, but only by the output of a detailed numerical computation of the internal structure of the underlying vacuum defect [85] 66] 67 68]. There is however an artificial mechanism (proceeding by a Kaluza Klein type projection of a simple Goto-Nambu model in an extended background with an extra space dimension) first suggested by Nielsen[75] that can easily be shown [4] to provide a particularly elegant illustration of the above formalism with a master function given (for an appropriate choice of the scaling of the stream function $\psi$ ) in terms of a constant mass parameter $m$ by

$$
\Lambda=\sqrt{m^{4}-m^{2} \chi}, \quad \tilde{\Lambda}=\sqrt{m^{4}-m^{2} \tilde{\chi}} .
$$

This has the rather exceptional but by no means unique property of providing an equation of state, for the relationship between $U$ and $T$, that has the same form in the magnetic regime $\chi>0>\tilde{\chi}$ 
as in the electric regime $\tilde{\chi}>0>\chi$. What is quite unique about this equation of state is its nondispersive - i.e. permanently transonic - "constant product" form (4.21), which can be seen 79 to be expressible parametrically in terms of a dimensionless self dual state function $\vartheta$ of the kind introduced in (5.20) by

$$
U=m^{2} \sqrt{1+\frac{\nu^{2}}{m^{2}}}=m^{2} \operatorname{coth} \vartheta, \quad T=m^{2} \sqrt{1-\frac{\mu^{2}}{m^{2}}}=m^{2} \tanh \vartheta,
$$

which gives

$$
c_{\mathrm{E}}=c_{\mathrm{L}}=\tanh \vartheta,
$$

so that by (4.21) and (4.20), the extrinsic ("wiggle") and longitudinal ("woggle") bicharacteristic vectors will coincide, having the form

$$
\ell_{ \pm}^{\mu}=u_{ \pm}^{\mu}=\cosh \vartheta u^{\mu} \pm \sinh \vartheta v^{\mu} .
$$

The revelation [4] of this unique transonicity property invalidates the too hasty claim 76 that Nielsen's elegant artifice 75] effectively represents the outcome in the "pure" string limit (in which effects of finite vortex thickness are neglected) of the Witten mechanism[7]. The latter requires a model [85] of generically dispersive type 31] that has been shown by the work of Peter 66] 676] 68] to be characterised typically by supersonicity, $c_{\mathrm{E}}>c_{\mathrm{L}}$. The elegant transonic model can nevertheless provide a crude but mathematically convenient approximation to a realistic description of a superconducting string that should be a considerable improvement not only on the use for this purpose of an unmodified Goto-Nambu model, but also on the more commonly used description provided by the naively linearised model [59 60] 601 62] 63 64 65 with equation of state given by constancy of the trace (5.3) which, by (4.10) and (4.20), is evidently characterised by permanent subsonicity, $c_{\mathrm{E}}<c_{\mathrm{L}}=1$.

In terms of the bicharacteristic vectors (5.51), the intrinsic equations of motion (5.19) can be recombined 13] [32] as an equivalent pair of divergence relations in the form

$$
\bar{\nabla}_{\rho}\left((U-T) \ell_{ \pm}^{\rho}\right)=-\ell_{\mp}^{\rho} \bar{f}_{\rho}
$$

which shows that the "left" and "right" moving "bicharacteristic currents", $(U-T) \ell_{+}{ }^{\rho}$ and $(U-$ $T) \ell_{-}{ }^{\rho}$ will each be conserved separately in the free case, i.e. when $\bar{f}_{\rho}$ vanishes. As an alternative presentation of the tangential force balance equations for this permanently transonic model, a little algebra suffices to show that the intrinsic equation of motion (5.22) can be rewritten for this case, in a form more closely analogous to that of the corresponding extrinsic equation of motion (5.23), as the pair of equations

$$
(U-T) \eta^{\mu}{ }_{\nu} \ell_{ \pm}{ }^{\rho} \bar{\nabla}_{\rho} \ell_{\mp}^{\nu}=\left(\eta^{\mu}{ }_{\nu}+\ell_{\mp}{ }^{\mu} \ell_{\mp} \nu\right) \bar{f}^{\nu}
$$


This tangentially projected part of the dynamic equations can now be recombined[79 with its orthogonally projected analogue (5.23), so as to give the complete set of force balance equations for the non dispersive permanently transcharacteristic string model (4.21) as the extremely useful pair of bicharacteristic propagation equations

$$
(U-T) \ell_{ \pm}{ }^{\rho} \bar{\nabla}_{\rho} \ell_{\mp}^{\mu}=\left(g^{\mu \nu}+\ell_{\mp}^{\mu} \ell_{\mp}^{\nu}\right) \bar{f}_{\nu} .
$$

The advantage of the characteristic formulation (5.54) is that it takes a particularly simple form when expressed in terms of the corresponding characteristic coordinates $\sigma^{ \pm}$on the worldsheet as defined by taking the "right moving" coordinate $\sigma^{+}$to be constant allong "left moving" characteristic curves, and taking the "left moving" coordinate $\sigma^{-}$to be constant along "right moving" characteristic curves, with the convention that the correspondingly parametrised bicharacteristic tangent vectors $\ell_{ \pm}^{\mu} \equiv \partial x^{\mu} / \partial \sigma^{ \pm}$should be future directed. In the case of a free motion (i.e. when the force term on the right of (5.54) vanishes) in a flat background, this simplification can be used 79 to obtain the complete solution of the dynamical equations in a very simple explicit form. The way this works is that (5.54) reduces just to

$$
\partial \ell_{ \pm}^{\nu} / \partial \sigma_{\mp}=0
$$

whose general solution is given in terms of a pair of generating curves $x_{ \pm}^{\mu}\{\sigma\}$ as a sum of single variable functions by the ansatz

$$
x^{\mu}=x_{+}^{\mu}\left\{\sigma^{+}\right\}+x_{-}^{\mu}\left\{\sigma^{-}\right\},
$$

which gives $\ell_{ \pm}^{\mu}=\dot{x}_{ \pm}^{\mu}$ using the dot here to denote the ordinary derivatives of the single variable functions with respect to the corresponding characteristic variables. The solution (5.56) generalises a result that is well known for more familiar but degenerate Goto Nambu case, in which the tangents to the generating curves are required to be null, $\ell_{ \pm}^{\mu} \ell_{ \pm} \mu=0$ (so that with the usual normalisation their space projections lie on what is known as the Kibble Turok sphere 87). The only restriction in the non - degenerate case is that they should be non-spacelike and future directed (the corresponding projections thus lying anywhere in the interior, not just on the surface, of a Kibble Turok sphere) the unit normalisation condition (5.21) being imposable as an option, not an obligation, by choosing the parameter $\sigma$ to measure proper time allong each separate generating curve.

This special property of being soluble by an ansatz of the same form (5.56) as has long been familiar for the Goto Nambu case can immediately be used to provide a new direct demonstration of the validity of the non-dispersive constant product model (4.21) for describing the average motion of a "wiggly" Goto Nambu string. The previously unpublished justification presented here is needed because my original argument 79] was merely of a qualitative heuristic nature, while Vilenkin's mathematical confirmation [80 was based on indirect energetic considerations, and has been called 
into question 31 on the grounds that it did not cover the most general class of wiggles that can be envisaged. It has recently been shown by Martin 88 that Vilenkin's method 81] (extending to the tension $T$ the concept of "renormalisation" that had previously been introduced for the energy density $U$ by Allen and Shellard 89 [90) can in fact be generalised straightforewardly so as to confirm the validity of (4.21) for all kinds of "wiggle" perturbations (subject only to the restriction that their amplitudes should not be so large as to bring about a significant rate of self intersection). Though based on manifestly muddled reasonning, the purported contrary demonstration 81 of higher order "deviations" from the constant product form (4.21) for the effective equation of state has left a residue of controversy and confusion. The need for an absolutely clear refutation of such allegations has motivated the formulation, as a more direct alternative to the energetic analysis developed by Vilenkin and Martin, of the new derivation presented here.

The new justification for the use of the elastic string model characterised by (4.21) consists simply of the observation that such a model implicitly underlies the diamond lattice discretisation that, since its original introduction by Smith and Vilenkin 91], has been commonly employed by numerical simulators 92 as a very convenient approximation scheme - of in principle unlimited accuracy - for the representation of a Goto-Nambu string worldsheet. As a way of replacing the exact continouous description by a discrete representation such as is necessary for numerical computation, the idea of the Smith Vilenkin method is simply to work with a pair of discrete sets of sampling points $x_{ \pm \mathrm{r}}^{\mu}=x_{ \pm}^{\mu}\left\{\sigma_{\mathrm{r}}\right\}$ determined by a corresponding discrete set of parameter values $\sigma_{\mathrm{r}}$ on the generating curves of the exact representation (5.56). This provides a "diamond lattice" of sample points given (for integral values of $\mathrm{r}$ and $\mathrm{s}$ ) by

$$
x_{\mathrm{rs}}^{\nu}=x_{+\mathrm{r}}^{\mu}+x_{-\mathrm{s}}^{\mu},
$$

that will automatically lie exactly on the "wiggly" Goto Nambu worldsheet (5.56), which is thus represented to any desired accuracy by choosing a sufficiently dense set of sampling parameter values $\sigma_{\mathrm{r}}$ on the separate "wiggly" null generating curves $x_{ \pm}^{\mu}\{\sigma\}$. The new remark I wish to make here is simply that the chosen set of sample points $x_{ \pm \mathrm{r}}^{\mu}=x_{ \pm}^{\mu}\left\{\sigma_{\mathrm{r}}\right\}$ on the separate "wiggly" null generators can also be considered to be sample points on a pair of smoothed out, and thus no longer null but timelike, interpolating curves that, according to the result 79 demonstrated above, can be interpreted according to (5.56) as generating a corresponding solution of the equations of motion for an elastic string model of the kind governed by (4.21). The not so "wiggly" elastic string worldsheet constructed by this smoothing operation will obviously be an even better approximation to the exact "wiggly" Goto Nambu worldheet than the original Smith Vilenkin lattice representation, which itself could already be made as accurate as desired by choosing a sufficiently high sampling resolution. No matter how far it is extrapolated to the future, the smoothed elastic string worldsheet generated according to (5.55) can never deviate significantly from the underlying "wiggly" Goto-Nambu worldsheet it is designed to represent because the exact worldsheet and the smoothed interpolation will always coincide precisely at each point of their shared Smith Vilenkin 
lattice (5.57). This highly satisfactory feature of providing a potentially unlimited accuracy could not be improved but would only be spoiled by any "deviation" from the originally proposed 79 form (4.21) for the effective equation of state.

After thus conclusively establishing that the permanently transonic elastic string model characterised by the simple constant product equation of state (4.21) (without any higher order corrections) provides an optimum description of the effect of microscopic wiggles in an underlying Goto Nambu model so long as self intersections remain unimportant (as was assumed in all the discussions 790 801 88 cited above), it remains to be emphasised that the neglect of such intersections will not be justified when the effective temperature 79 82 of the wiggles is too high (as will presumably be the case[13] during a transient period immediately following the string forming phase transition). The result of such intersections will be the formation of microscopic loops, of which some will subsequently be reconnected, but of which a certain fraction will escape. Estimation of the dissipative cooling force density that would be needed to allow for such losses remains a problem for future work.

\section{Symmetric Configurations including Rings and their Cos- mological Implications}

Whenever the background space time metric is invariant under the action of a (stationarity, axisymmetry, or other) continuous invariance group generated by a solution $k^{\mu}$ Killing equation (2.31), i.e. in the notation of $(2.20)$

$$
\overrightarrow{k \mathcal{E}} g_{\mu \nu}=0
$$

then any string that is isolated (i.e. not part of the boundary of an attached membrane) will have a corresponding momentum current (interpretable, depending on the kind of symmetry involved, as representing a flux of energy, angular momentum, or whatever) given by

$$
\bar{P}^{\mu}=\bar{T}_{\nu}^{\mu} k^{\nu}
$$

In accordance with (2.33), this will satisfy a source equation of the form

$$
\bar{\nabla}_{\mu} \bar{P}^{\mu}=\bar{f}_{\mu} k^{\mu},
$$

which means that the corresponding flux would be strictly conserved when the string were not just isolated but free, i.e. if the background force $\bar{f}_{\mu}$ were zero. When the string is subject to a background force of the Lorentz-Joukowsky form (5.33) that arises from background electromagnetic and Kalb-Ramond fields, then provided these background fields are also invariant under the symmetry group action generated by $k^{\mu}$, i.e. in the notation of $(2.20)$

$$
\overrightarrow{k \mathcal{E}} A_{\mu}=0, \quad \overrightarrow{k \mathcal{E}} B_{\mu \nu}=0
$$


it can be seen to follow that, although the physically well defined surface current $\bar{P}^{\mu}$ will no longer be conserved by itself, it still forms part of a gauge dependent generalisation, $\overline{\mathcal{P}}^{\mu}$ say, that is strictly conserved,

$$
\bar{\nabla}_{\mu} \overline{\mathcal{P}}^{\mu}=0,
$$

and that is given, in terms of the gauge dependent generalisation

$$
\overline{\mathcal{T}}^{\mu}{ }_{\nu}=\bar{T}^{\mu}{ }_{\nu}+\bar{J}^{\mu} A_{\mu}+\bar{W}^{\mu \rho} B_{\nu \rho}
$$

of the surface stress momentum energy density tensor, by

$$
\overline{\mathcal{P}}^{\mu}=\overline{\mathcal{T}}^{\mu}{ }_{\nu} k^{\nu}=\bar{P}^{\mu}+\left(e c^{\mu} A_{\nu}+\kappa \mathcal{E}^{\mu \rho} B_{\nu \rho}\right) k^{\mu} .
$$

The purpose of this section is to consider string configurations that share the background symmetry under consideration. A configuration that is symmetric in this sense will be characterised by the condition

$$
\perp_{\nu}^{\mu} k^{\nu}=0,
$$

meaning that the symmetry generator $k^{\mu}$ is tangential to the worldsheet, and the corresponding Lie invariance condition on its surface stress momentum energy density tensor will have the form

$$
k^{\mu} \nabla_{\mu} \bar{T}^{\nu \rho}=2 \bar{T}^{\mu(\nu} \nabla_{\mu} k^{\rho)} .
$$

Under such conditions, as well as the ordinary momentum flux $\bar{P}^{\mu}$, what may be termed the adjoint momentum flux,

$$
{ }^{\dagger} \bar{P}^{\mu}=k^{\nu \dagger} \bar{T}_{\nu}{ }^{\mu}, \quad{ }^{\dagger} \bar{T}_{\nu}{ }^{\mu}=\mathcal{E}_{\nu \rho} \mathcal{E}^{\mu \sigma} \bar{T}_{\sigma}^{\rho},
$$

will also obey an equation of the form (6.3), i.e.

$$
{ }^{\dagger} \bar{\nabla}_{\mu} \bar{P}^{\mu}=\bar{f}_{\mu} k^{\mu},
$$

and hence would also be conserved if the string were free. In the presence of an electromagnetic or Kalb Ramond background, it can be seen that, like the ordinary momentum flux, this adjoint momentum flux has a gauge dependent extension,

$$
\overline{\mathcal{P}}^{\mu}=k^{\nu \dagger} \overline{\mathcal{T}}_{\nu}{ }^{\mu}, \quad \dagger \overline{\mathcal{T}}_{\nu}{ }^{\mu}=\mathcal{E}_{\nu \rho} \mathcal{E}^{\mu \sigma} \overline{\mathcal{T}}^{\rho}{ }_{\sigma},
$$

that will share with the generalised momentum flux $\overline{\mathcal{P}}^{\mu}$ (that would be conserved even if the string did not share the symmetry of the background) the property of obeying a strict surface current conservation law, namely

$$
\bar{\nabla}_{\mu} \overline{\mathcal{P}}^{\mu}=0 .
$$

The group invariance conditions

$$
k^{\nu} \nabla_{\nu} p_{\mu}+p_{\nu} \nabla_{\mu} p^{\nu}=0, \quad k^{\nu} \nabla_{\nu} \tilde{p}_{\mu}+\tilde{p}_{\nu} \nabla_{\mu} p^{\nu}=0,
$$


that are the analogues of (6.9) for the separate mutually dual pair of internal momentum covectors $\tilde{p}_{\mu}$ and $p_{\mu}$ associated with the internal current within the string, can be rewritten, with the aid of the corresponding electromagnetic background invariance condition (6.4), in the form

$$
k^{\rho} \mathcal{E}^{\mu \nu}\left(\nabla_{\nu} \tilde{p}_{\mu}+\frac{e}{2} F_{\nu \rho}\right)=\mathcal{E}^{\rho \nu} \nabla_{\nu} \omega, \quad k^{\rho} \mathcal{E}^{\mu \nu} \nabla_{\nu} p_{\mu}=\mathcal{E}^{\rho \nu} \nabla_{\nu} \beta,
$$

where $\omega$ and $\beta$ are are the Bernoulli type scalars given by

$$
\omega=\tilde{\beta}+e A_{\mu} k^{\mu}, \quad \tilde{\beta}=\tilde{p}_{\mu} k^{\mu}, \quad \beta=p_{\mu} k^{\mu} .
$$

If $k^{\mu}$ is timelike, the corresponding symmetry will be interpretable as stationarity, while the more restrictive case 993 in which the string is actually static (in the sense that there is no transverse current component relative to the background rest frame determined by $k^{\mu}$ ) will be given by the condition that the second Bernouilli constant, $\beta$ should vanish.

It can be seen from (6.15) that the internal equations of motion (5.39) and (5.41) are equivalent in this group invariant case simply to the corresponding pair of Bernouilli type conservation laws to the effect that $\omega$ and $\beta$ (but, unless the electromagnetic field is absent, not $\tilde{\beta}$ ) should both be constant over the worldsheet. This observation allows the problem of solving the dynamical for a symmetric configuration of the kind of ("perfectly elastic", i.e. barocentric) string model under consideration to one of solving just the extrinsic equations governing the location of the worldsheet. A recent investigation 94 based on the systematic use of variational methods in the restricted case for which the Kalb-Ramond coupling was absent has drawn attention to the interest of extrapolating the Bernoulli constants outside the supporting worldsheet as a pair of scalar fields defined over the entire background spacetime by the uniformity conditions

$$
\nabla_{\mu} \omega=0, \quad \nabla_{\mu} \beta=0,
$$

and formulating the problem in terms a certain particular worldsheet tangent vector that is defined by

$$
X^{\mu}=\mathcal{E}^{\mu \nu \dagger} \bar{P}_{\nu}=k^{\nu} \mathcal{E}_{\nu \rho} \bar{T}^{\rho \mu} .
$$

This vector will be expressible in terms of the variables introduced in previous section as

$$
X^{\mu}=\frac{\beta \Lambda}{\chi} c^{\mu}+\frac{\tilde{\beta} \tilde{\Lambda}}{\tilde{\chi}} \tilde{c}^{\mu},
$$

while the Killing vector itself will be expressible in analogous form by

$$
k^{\mu}=\frac{\tilde{\beta}}{\Lambda-\tilde{\Lambda}} c^{\mu}+\frac{\beta}{\tilde{\Lambda}-\Lambda} \tilde{c}^{\mu} .
$$

The latter gives for the (real or pure imaginary) Killing vector amplitude, $V$ say (which in the case of stationary symmetry, for which it is real, will be interpretable as an effective gravitational 
potential field), an expression of the form

$$
V^{2}=-k^{\mu} k_{\mu}=\frac{\beta^{2}}{\chi}+\frac{\tilde{\beta}^{2}}{\tilde{\chi}},
$$

that, in view of (6.16), and of the state functional relationship between $\chi$ and $\tilde{\chi}$, can be solved for any particular choice of the constant "tuning parameters" $\omega$ and $\beta$ to determine the internal variables $\chi$ and $\tilde{\chi}$ as functions of the (gravitational type) potential $V$ and the (electric type) potential $A_{\mu} k^{\mu}$, and hence by implication as scalar fields over the entire background space, not just on the worldsheet where they were originally defined. In a similar manner, it can be seen that the contraction of the worldsheet generating vector $X^{\mu}$ with the Killing vector field will be given simply by

$$
k^{\mu} X_{\mu}=\beta \tilde{\beta},
$$

and that its (real or pure imaginary) amplitude, $X$ say, will given by an expression of the form

$$
X^{2}=X^{\mu} X_{\mu}=\frac{\beta^{2} \Lambda^{2}}{\chi}+\frac{\tilde{\beta}^{2} \tilde{\Lambda}^{2}}{\tilde{\chi}}
$$

whereby it too is implicitly defined as a function of $V$ and $A_{\mu} k^{\mu}$, and hence as a well defined scalar field, not just on the worldsheet but, by (6.16), also over the background as a whole, its gradient being given by

$$
X \nabla_{\mu} X=\Lambda \tilde{\Lambda} V \nabla_{\mu} V+e \tilde{\beta}(\Lambda-\tilde{\Lambda}) \frac{\tilde{\Lambda}}{\tilde{\chi}} \nabla_{\mu}\left(A_{\nu} k^{\nu}\right) .
$$

Previous experience 94 with the case in which only the electromagnetic but not the Magnus force contribution is present suggests the interest of formulating the problem in terms of the propagation of the special generating vector $X^{\mu}$, which (using the formulae (5.5) and (5.43) of the previous section) can be seen from (6.19) and (6.20) to be given by

$$
X^{\nu} \nabla_{\nu} X_{\mu}=\Lambda \tilde{\Lambda} k^{\nu} \nabla_{\nu} k_{\mu}+X^{\nu} \mathcal{E}_{\nu \rho} k^{\rho} \bar{f}_{\mu},
$$

where $\bar{f}^{\mu}$ is the background force as given by (5.33). It can now be seen that the two preceeding equations can be combined to give the equation of motion for the worldsheet generating vector $X^{\mu}$ in the very elegant and convenient final form

$$
X^{\nu} \nabla_{\nu} X_{\mu}-X \nabla_{\mu} X=\mathcal{F}_{\mu \nu} X^{\nu}
$$

in terms of a pseudo Maxwellian field given by

$$
\mathcal{F}_{\mu \nu}=e \beta F_{\mu \nu}+\kappa N_{\mu \nu \rho} k^{\rho}=2 \nabla_{[\nu} \mathcal{A}_{\rho]},
$$

where $\mathcal{A}_{\mu}$ is a gauge dependent pseudo-Maxwellian potential covector given by

$$
\mathcal{A}_{\mu}=e \beta A_{\mu}+\kappa B_{\mu \nu} k^{\nu} .
$$


If $k^{\mu}$ is timelike so that the corresponding symmetry is interpretable as stationarity, then the equation of motion (6.26) will be interpretable as the condition for the string to be in equilibrium with the given values of the constant "tuning" parameters $\omega$ and $\beta$, (of which, as remarked above, the latter, $\beta$, will vanish in the case of an equilibrium that is not just stationary but static 93). The new result here is that the Joukowski type "lift" force (which was not allowed for in the previous analysis 94]) due to the Magnus effect on the string, as it "flies" (like an aerofoil) through the background medium represented by the current 3 -form $N_{\mu \nu \rho}$, has just the same form as an extra Lorentz type electromagnetic (indeed in the stationary case purely magnetic) force contribution.

When the genuine electromagnetic background coupling, and the similarly acting Kalb Ramond coupling are both absent, then (as pointed out previously 94]) the equation of motion (6.26) (that is the equilibrium condition for "steady flight" in the stationary case) is just a simple geodesic equation with respect to, not the actual background spacetime metric $g_{\mu \nu}$, but the conformally modified metric $X^{2} g_{\mu \nu}$, with the conformal factor $X^{2}$ determined as a field over the background by (6.23) in conjunction with (6.17) and (6.21). Even when the Lorentz and Joukowski force contributions are present, the equation (6.26) governing the propagation of the world sheet generator $X^{\mu}$ retains a particularly convenient Hamiltonian form, given by

$$
X^{\mu}=\frac{d x^{\mu}}{d \sigma}=\frac{\partial H}{\partial \Pi_{\mu}}, \quad \frac{d \Pi_{\mu}}{d \sigma}=-\frac{\partial H}{\partial x^{\mu}},
$$

for the quadratic Hamiltonian function

$$
H=\frac{1}{2} g^{\mu \nu}\left(\Pi_{\mu}-\mathcal{A}_{\mu}\right)\left(\Pi_{\nu}-\mathcal{A}_{\nu}\right)-\frac{1}{2} X^{2},
$$

subject to a restraint fixing the (generically non-affine) parametrisation $\sigma$ of the trajectory by the condition that the numerical value of the Hamiltonian (which will automatically be a constant of the motion) should vanish,

$$
H=0,
$$

together with a further momentum restraint, determining the (automatically conserved) relative transport rate $\Pi_{\mu} k^{\mu}$ in accordance with the relation (6.22) by the condition

$$
\Pi_{\mu} k^{\mu}=\omega \beta .
$$

The Hamiltonian momentum covector itself can be evaluated as

$$
\Pi_{\mu}=X_{\mu}+\mathcal{A}_{\mu}=\mathcal{E}_{\mu \nu}^{\dagger} \overline{\mathcal{P}}^{\nu}
$$

or more explicitly, in terms of the original conserved generalised momentum flux $\overline{\mathcal{P}}^{\mu}$ as given by (6.7), and the gradients of the scalar (stream function and phase) potentials introduced in (5.28) and (5.42), as

$$
\Pi_{\mu}=k^{\nu} \mathcal{E}_{\nu \rho} \overline{\mathcal{T}}^{\rho}{ }_{\mu}=\overline{\mathcal{P}}^{\nu} \mathcal{E}_{\nu \mu}+\omega \nabla_{\mu} \psi+\beta \nabla_{\mu} \varphi
$$


The advantage of a Hamiltonian formulation is that it allows the problem to be dealt with by obtaining the momentum covector in the form $\Pi_{\mu}=\nabla_{\mu} S$ from a solution of the corresponding Hamilton Jacobi equation, which in this case will take the form

$$
g^{\mu \nu}\left(\nabla_{\mu} S-\mathcal{A}_{\mu}\right)\left(\nabla_{\nu} S-\mathcal{A}_{\nu}\right)=X^{2},
$$

with $X^{2}$ given by (6.23) via (6.21), while the restraint (6.32) gives the condition

$$
k^{\nu} \nabla_{\nu} S=\beta \omega .
$$

Generalising results obtained previously 95 [96] for the Goto Nambu limit case, it has recently been shown 94 that for a string model of the non-dispersive permanently trasonic type with the constant product equation of state (4.21) that is governed by the Lagrangian (5.48) (as obtained [4] both from the Nielsen dimensional reduction mechanism and also 79], as explained in Section 5, from the more physically realistic "wiggly" string approximation) the stationary Hamilton Jacobi equation is exactly soluble by separation of variables in a Kerr black hole spacetime, not just of the ordinary asymptotically flat kind but even of the generalised asymptotically De Sitter kind 97 98. Except in the Schwarschild-De Sitter limit, where it could of course have been predicted as a consequence of spherical symmetry, this separability property still seems rather miraculous, reflecting a "hidden symmetry" of the Kerr background that is still by no means well understood. The newly discovered separability property [94] is not just an automatic consequence of the simpler, though when first discovered already surprising, property of separability for the ordinary geodesic equation [99] but depends on a more restrictive requirement of the kind needed for the more delicate separability property of the scalar wave equation 100. (It is however more robust than the separability properties that have turned out to hold for higher spin bosonic 101 and fermionic 102 103 [104 wave equations, and other related systems 105 106.)

The most cosmologically important application of the formalism that has just been presented is to the equilibrium of small closed string loops in the mathematically relatively trivial case for which the background gravitational, electromagnetic, and Kalb-Ramond fluid are negligible, so that the trajectories generated by solutions of (6.26) will all just be straight lines in a Minkowski background. Since in such a background the Killing vector trajectories are also straight in the case of stationarity (though not of course for axisymmetry) this might at first be perceived implying that the worldsheet of a stationary string in an empty Minkowski background would necessarily be flat. This conclusion would exclude the possibility of closed loop equilibrium states in the absence of a background field, and indeed cosmologists seem (albeit for other reasons) to have entirely overlooked the possibility that such states might exist until the comparitively recent publication of an epoch making paper by Davis and Shellard[107] provided the first counterexamples (the only previously considered equilibrium states [108] 61] 62] 63] having been based on a magnetic support mechanism that was was finally judged to be too feeble to be effective except 111 as a minor correction). 
The loophole in the deduction that if the trajectories generated by $X^{\mu}$ and by $k^{\mu}$ are both straight then the worldsheet must be flat is that it is implicitly based on the assumption that the two kinds of trajectories cross each other transversly. However there will be no restriction on the curvature in the transverse direction in the critical case for which the two kinds of trajectory coincide, i.e. for which $X^{\mu}$ and $k^{\mu}$ are parallel. The condition for criticality in this sense is expressible as

$$
X^{\mu} \mathcal{E}_{\mu \nu} k^{\nu}=0,
$$

which can be seen from the original definition (6.18) to be interpretable as meaning that the tangent covector $\chi_{\mu}=\mathcal{E}_{\mu \nu} k^{\nu}$ satisfies the extrinsic characteristic equation (3.10), or in other words that the killing vector $k^{\mu}$ itself is bicharacteristic, in the sense of being directed allong the propagation direction of extrinsic perturbations of the world sheet. The criticality condition (6.37) is thus interpretable as a condition of characteristic flow. It means that the "running velocity", $v$ say, of relative motion of the intrinsicly preferred rest frame of the string (as determined by whichever of $c^{\mu}$ and $\tilde{c}^{\mu}$ is timelike) relative to the background frame specified by the (in this case necessarily timelike) Killing vector $k^{\mu}$ is the same as the extrinsic propagation velocity $c_{\mathrm{E}}$ given by (4.10).

In the presence of generic gravitational, electromagnetic and Kalb Ramond forces, the criticality condition (6.37) can be satisfied at particular positions, such as where there is a transition from a subcharacteristic running velocity, $v<c_{\mathrm{E}}$ to a supercharacteristic running velocity $v>c_{\mathrm{E}}$ (as will occur for instance on a string in a steady state of radial flow into a Schwarzschild black hole) or where there is a cusp, with $v=c_{\mathrm{E}}$ but with subcharacteristic flow $v<c_{\mathrm{E}}$ on both sides. However in view of the constancy of the Bernoulli "tuning" parameters $\omega$ and $\beta$, the absence of any background field will always allow, and generically (the special integrable case (5.49) being an exception) will ensure, uniformity of the state of the string, so that the transcharacteristic flow condition (6.37) can be satisfied throughout its length. The space configuration of such a uniformly transcharacteristic steady string state can have arbitrarily variable curvature, and so is compatible with a closed loop topology.

The question of closed loop equilibrium states did not arise in the earliest studies 110 of cosmic strings, which were restricted to the Goto-Nambu model whose bicharacteristics are always null and so can never be aligned with the timelike Killing vector generating a stationary symmetry. However in a generic string model[30] for which the 2-dimensional longitudinal Lorentz of the internal structure is broken by a current, whether of the neutral kind exemplified in an ordinary violin string or the electromagnetic kind exemplified 85 66 67 67 68 by Witten's superconducting cosmic string model, the bicharacteristic directions will generically be timelike 34 (spacelike bicharacteristics being forbidden by the requirement of causality) so there will be no obstacle to their alignement with a timelike Killing vector in accordance with the criticality condition (6.37), i.e. to having a running velocity given by $v=c_{\mathrm{E}}$. The simple "toy" complex scalar field model on which the pionneering cosmic string studies [5] were based had longitudinally Lorentz invariant vortex defects 
(of "local" or "global" type depending on the presence or absence of coupling to a gauge field) that were describable at a microscopic level by string models (with Kalb Ramond coupling in the "global" case) that were indeed of the special Goto-Nambu type. However the extra degrees of field freedom (starting with the additional scalar field introduced by Witten in his original superconducting example []) that are needed in successively more realistic models 111 112 make it increasingly difficult to avoid the formation of internal structure breaking the longitudinal Lorentz invariance and reducing the extrinsic characteristic velocity to the subluminal range $c_{\mathrm{E}}<1$ at which stationary equilibrium with the critical running velocity $v=c_{\mathrm{E}}<1$ becomes possible.

The cosmological significance of this is that whereas Goto Nambu string loops cannot ultimately avoid gravitationally or otherwise radiating away all their energy 113 114 115, since they have no equilibrium states into which they might settle down, on the other hand more general kinds of strings, whose occurrence would now seem at least as plausible 111 112, can leave a relic distribution of stationary loop configurations that may survive indefinitely 107] [116] 117] [118]. One would expect such configurations to be those that minimise the energy for given values of the relevant globally conserved quantities, of which there might be a considerable number in the more complicated multiply conducting models that might be considered, but of which there are only a single pair in the "barotropic" type string considered here, namely the stream function winding number $\oint d \psi$ and the phase winding number $\oint d \varphi$ whose respective constancy results from the conservation of the mutually dual pair of currents $c^{\mu}$ and $\tilde{c}^{\mu}$.

The potential cosmological importance of such a distribution of relic loops was first pointed out by Davis and Shellard 107 116 117, who emphasised that in the case of the "heavy" cosmic strings whose existence had been postulated to account for galaxy formation, the ensuing relics, even if formed with very low efficiency, would be more that sufficient to give rise to a catastrophic cosmological mass excess of the kind first envisaged as arising from the formation of monopoles. According to a more recent and detailed order of magnitude estimate of my own 118 , the dimensionless coupling constant $\mathrm{Gm}^{2} \approx 10^{-6}$ characterising "heavyweight" strings, meaning those arising from G.U.T. symmetry breaking (for which the relevant Higgs mass scale $m$ is within a factor of order a thousand of the Plank mass) would have to be reduced below a value given very roughly by $G m^{2} \approx 10^{-26}$ to avoid a cosmological mass excess today. This estimate 118 (based on considerations of the kind discussed during the present meeting by Zurek) should be regarded as provisional, pending the more deeper investigation that would seem to be needed. What is remarkable about this tentative limit is that it corresponds to a Higgs mass scale $m$ of roughly the same order as that at which electroweak symmetry breaking is believed to occur, which is rather suggestive in view of the fact that although the "standard" electroweak model does not give rise to stable string like vortex defects 119, nevertheless such defects do occur in many of its most commonly considered competitors 111 .

The cosmological implications (as an argument against "heavy" cosmic string formation, or 
more positively as source of "dark matter" in the form of "lightweight" string loops due to electroweak symmetry breaking) of the long term survival of cosmic string loops, motivates more thorough investigation of equilibrium states that may be involved, a particularly important question being that of their stability. Prior to the derivation of the general symmetric string generator equation (6.26), the only closed loop equilibrium states to have been considered were the circular kind referred to by Davis and Shellard 107] 116] 117] as "vortons" a term that is more appropriate for axisymmetric ring states in the "global" case than in the gauge coupled "local" case for which the string description is most accurate. The first general investigation 1120 of such circular ring states showed that under conditions of purely centrifugal support (neglecting possible electromagnetic corrections of the kind evaluated more recently 109]) the condition (6.37) for equilibrium, namely the requirement of a transcharacteristic rotation speed $v=c_{\mathrm{E}}$, is such that the ring energy is minimised with respect to perturbations preserving the circular symmetry. However a more recent investigation of non-axisymmetric perturbations has shown that although there are no unstable modes for states of subsonic rotation [69] (as exemplified by a cowboy's lassoe loop) with $v=c_{\mathrm{E}}<c_{\mathrm{L}}$, instability can nevertheless occur for rotation in the supersonic regime [70 that (contrary to what was implicitly assumed in earlier work 59 60] 61 62] 63] 64 65] using the subsonic type of model given by a linear equation of state for which the sum $U+T$ is constant) has been shown by Peter 66 67 68 to be relevant in the kind of cosmic vortex defects that have been considered so far. Although the first category of string loop equilibrium states to have been studied systematically has been that of circular ring configurations 120, it has been made clear by recent work 94 69 that, as explained above, arbitrary non circular equilibrium states are also possible. The stability such more general equilibrium states has not yet been investigated, carried out, but it seems plausible that while some of them may be destroyed by the recently discovered classical instability mechanism[69] [70], and also the kind of quantum tunnelling instability mechanism considered by Davis 116, it does not seem likely that such mechanisms could be so consistently efficient as to prevent the long term survival of a lot of other stationary loop states.

\section{Acknowledgements.}

I wish to thank B. Allen, C. Barrabès, U. Ben-Ya'acov, A-C. Davis, R. Davis, V. Frolov, G. Gibbons, R. Gregory, T. Kibble, K. Maeda, X. Martin, P. Peter, T. Piran, D. Polarski, M. Sakellariadou, P. Shellard, P. Townsend, N. Turok, T. Vachaspati, and A. Vilenkin, for many stimulating or clarifying discussions.

\section{References}

[1] P.A.M. Dirac, Proc. Roy. Soc. London A268, 57 (1962). 
[2] P.S. Howe, R.W. Tucker, J. Phys. A10, L155 (1977).

[3] A. Achúcarro, J. Evans, P.K. Townsend, D.L. Wiltshire, Phys. Lett. 198 B, 441 (1987).

[4] B. Carter, in Formation and Evolution of Cosmic Strings, ed. G. Gibbons, S. Hawking, T. Vachaspati, pp143-178 (Cambridge U.P., 1990).

[5] T.W.B. Kibble, J.Phys. A9, 1387 (1976).

[6] A. Vilenkin, A.E. Everett, Phys. Rev. Lett 48, 1867 (1982).

[7] E. Witten, Nucl. Phys. B249, 557 (1985).

[8] B. Carter, J. Geom. Phys. 8, 53 (1992).

[9] B. Carter, J. Class. Quantum Grav. 9, 19 (1992).

[10] L.P. Eisenhart, Riemannian Geometry (Princeton U.P., 1926, reprinted 1960).

[11] J. Stachel, Phys. Rev. D21, 2171 (1980).

[12] J.A. Schouten, Ricci Calculus (Springer, Heidelberg, 1954).

[13] B. Carter, M. Sakellariadou, X. Martin, Phys. Rev. D50, 682 (1994)

[14] A. Vilenkin, Phys. Rev. D43, 1060 (1991).

[15] R. Penrose, W. Rindler, Spinors and Space-Time (Cambridge U.P., 1984).

[16] I. Bars, C.N. Pope, Class. Quantum Grav., 5, 1157 (1988).

[17] P. Sikivie, Phys. Rev. Lett. 48, 1156 (1982).

[18] A. Vilenkin, A.E. Everett,Phys. Rev. Lett. 48, 1867 (1982).

[19] E.P.S. Shellard, in Formation and Evolution of Cosmic Strings, ed. G. Gibbons, S. Hawking, T. Vachaspati, pp107-115 (Cambridge U.P., 1990).

[20] Y. Nambu, Nucl. Phys. B130, 505 (1977).

[21] N.S. Manton Phys. Rev. D28, 2019 (1983).

[22] T. Vachaspati, A. Achúcarro, Phys. Rev. D44, 3067 (1991).

[23] T. Vachaspati, M. Barriola, Phys. Rev. Letters 69, 1867 (1992). 
[24] A. Dabholkar, J.M. Quashnock, Nucl. Phys. B333, 815 (1990).

[25] R.A. Battye, E.P.S. Shellard, "Global string radiation", DAMTP preprint (Cambridge, 1994).

[26] B. Carter, Class. Quantum Grav. 11, 2013 (1994).

[27] A. Vilenkin, T. Vachaspati, Phys. Rev. D35, 1138 (1987).

[28] R.L. Davis, E.P.S. Shellard, Phys. Rev. Lett. 63, 2029 (1989).

[29] M. Sakellariadou, Phys. Rev. D44, 3767 (1991).

[30] U. Ben-Ya'acov, Nucl. Phys. B382, 597 (1992).

[31] B. Carter, Phys. Lett. B224, 61 (1989).

[32] A.L. Larsen, Class. Quantum Grav. 10L, 35 (1993).

[33] J. Garriga, M. Sakellariadou, Phys. Rev. 48, 2502 (1993).

[34] B. Carter, Phys. Lett. B228, 466 (1989).

[35] B. Carter, Phys. Rev. 48, 4835 (1993)

[36] J. Garriga, A. Vilenkin, Phys. Rev. D47, 3265 (1993).

[37] A.L. Larsen, V. Frolov, Nucl. Phys. B414, 129 (1994).

[38] J. Garriga, A. Vilenkin, Phys. Rev. D44, 1007 (1991).

[39] J. Garriga, A. Vilenkin, Phys. Rev. D45, 3469 (1992).

[40] J. Guven, Phys. Rev. D48, 4464 (1993).

[41] J. Guven, Phys. Rev. D48, 5563 (1993).

[42] B. Carter, Proc. Roy. Soc. Lond. A372, 169 (1980).

[43] A. Polyakov, Nucl. Phys. B268, 406 (1986).

[44] K.I. Maeda, N. Turok, Phys. Lett. B202, 376 (1988).

[45] R. Gregory, Phys. Lett. B206, 199 (1988).

[46] R. Gregory, Phys. Rev. D43, 520 (1993).

[47] D. Garfinkle, R. Gregory, Phys. Rev. D41 1889 (1990). 
[48] R. Gregory, D. Haws, D. Garfinkle, Phys. Rev. D42 343 (1991).

[49] V. Silveira, M.D. Maia, Phys. Lett A174, 280 (1993).

[50] C. Barrabès, B. Boisseau, M. Sakellariadou, Phys. Rev. D49, 2734 (1994).

[51] B. Carter, R. Gregory, Curvature corrections to dynamics of domain walls, DAMTP preprint (Cambridge, 1994).

[52] P.S. Letelier, Phys. Rev. D41, 1333 (1990).

[53] D. H. Hartley, R.W. Tucker, in Geometry of Low Dimensional Manifolds, 1 ( L.M.S. Lecture Note Series 150, ed. S. Donaldson, C. Thomas (Cambridge U.P., 1990).

[54] H. Arodz, A. Sitarz, P. Wegrzyn, Act. Phys. Polon. B, 22, 495 (1991); 23, 53 (1992).

[55] B. Boisseau, P.S. Letelier, Phys. Rev. D46, 1721 (1992).

[56] B. Carter, Equations of motion of a stiff geodynamic string or higher brane, Meudon preprint (to appear in Class. Quantum Gravity 11, 1994).

[57] S.W. Hawking, G.F.R. Ellis, The Large Scale Structure of Spacetime (Cambridge U.P., 1973).

[58] B. Carter, in Active Galactic Nuclei, eds. C. Hazard, S.Mitton, pp 273-300 (Cambridge U. P., 1976).

[59] D.N. Spergel, T. Piran, J. Goodman, Nucl. Phys. B291, 847 (1987).

[60] A. Vilenkin, T. Vachaspati, Phys. Rev. Lett. 58, 1041 (1987)

[61] E. Copeland, M. Hindmarsh, N. Turok, Phys. Rev. Lett. 58, 1910 (1987)

[62] E. Copeland, D. Haws, M. Hindmarsh, N. Turok, Nucl. Phys. B306, 908 (1988).

[63] D. Haws, M. Hindmarsh, N. Turok, Phys. Lett. B209, 225 (1988).

[64] D.N. Spergel, W.H. Press, R.J. Scherrer, Phys. Rev. D39, 379 (1989)

[65] P. Amsterdamski, Phys Rev. D39, 1534 (1989).

[66] P. Peter, Phys. Rev. D45, 1091 (1992). 
[67] P. Peter, Phys. Rev. D46, 3335 (1992).

[68] P. Peter, Phys. Rev. D47, 3169 (1993).

[69] B. Carter, X. Martin, Ann. Phys. 227, 151 (1993).

[70] X. Martin, Meudon preprint (to appear in Phys. Rev. D, 1994).

[71] R.L. Davis, E.P.S. Shellard, Phys. Lett B209, 485 (1988).

[72] R.L. Davis, E.P.S. Shellard, Nucl. Phys. B323 209 (1989)

[73] B. Carter, Phys. Lett. B238, 166 (1990).

[74] P. Peter, Phys. Lett. B298, 60 (1993).

[75] N.K. Nielsen, Nucl. Phys. B167, 248 (1980).

[76] N.K. Nielsen, P. Olesen, Nucl. Phys. B291, 829 (1987).

[77] A. Davidson, A.K. Wali, Phys. Lett. B213, 419 (1988).

[78] A. Davidson, A.K. Wali, Phys. Rev. Lett. 61, 1450 (1988).

[79] B. Carter, Phys. Rev. D41, 3869 (1990).

[80] A. Vilenkin, Phys. Rev. D41, 3038 (1990).

[81] J.Hong, J.Kim, P. Sikivie, Phys. Rev. Lett. 69, 2611 (1980).

[82] B. Carter, Nucl. Phys. B412, 345 (1994).

[83] E. Witten, Phys. Lett. B158, 243 (1985).

[84] R.L. Davis, E.P.S. Shellard, Phys. Lett. B214, 219 (1988).

[85] A. Babul, T. Piran, D.N. Spergel, Phys. Lett. B202, 207 (1988).

[86] D.N. Spergel, W.H. Press, R.J. Scherrer, Phys. Rev. D39, 379 (1989).

[87] T.W.B. Kibble, N. Turok, Phys. Lett. B116, 141 (1982).

[88] X. Martin, Meudon preprint (1994).

[89] B. Allen, E.P.S. Shellard, Phys. Rev. Lett. 64, 119 (1990). 
[90] E.P.S. Shellard, B. Allen, in Formation and Evolution of Cosmic Strings, ed. G. Gibbons, S. Hawking, T. Vachaspati, pp421-448 (Cambridge U.P., 1990).

[91] A.G. Smith, A. Vilenkin, Phys. Rev. D36, 990 (1987).

[92] A. Albrecht, in Formation and Evolution of Cosmic Strings, ed. G. Gibbons, S. Hawking, T. Vachaspati, pp403-419 (Cambridge U.P., 1990).

[93] B. Carter, Class. and Quantum Grav. 7, L69 (1990).

[94] B. Carter, V.P. Frolov, O. Heinrich, Class. and Quantum Grav. 8, 135 (1991).

[95] V.P. Frolov, V.D. Skarzhinsy, A.I. Zelnikov, O. Heinrich, Phys. Lett. 224, 225 (1989).

[96] B. Carter, V.P. Frolov, Class. Quantum Grav. 6, 569 (1989).

[97] B. Carter, in Black Holes (Les Houches, 1972), ed. C. and B.S. DeWitt, pp 57-214 (Gordon and Breach, New York, 1973).

[98] G.W. Gibbons, S.W. Hawking, Phys. Rev. D15, 2738 (1976).

[99] B. Carter, Phys. Rev. 174, 1559 (1968).

[100] B. Carter, Commun. Math. Phys. 99, 563 (1968).

[101] S.A. Teukolsky, Astroph. J. 185, 283 (1973).

[102] W. Unruh, Phys. Rev. Lett. 31, 1265 (1973).

[103] S. Chandrasekhar, Proc. Roy. Soc. Lond. A349, 571 (1976).

[104] R. Guven, Phys. Rev. D22, 2327 (1980).

[105] J-A. Marck, Proc. Roy. Soc. Lond. A385, 431 (1983).

[106] B. Carter, J. Math. Phys. 28, 1535 (1987).

[107] R.L. Davis, E.P.S. Shellard, Phys. Lett. B209, 485 (1988).

[108] J. Ostriker, C. Thompson, E. Witten, Phys. Lett B180, 231 (1986)

[109] P. Peter, Phys. Lett. B298, 60 (1993).

[110] T.W.B. Kibble, Phys. Rep. 67, 183 (1980). 
[111] P. Peter, Phys. Rev., D46, 3322 (1992).

[112] W. B. Perkins, A-C. Davis, Nucl. Phys. B406, 377 (1993).

[113] N. Turok, Nucl. Phys. B242, 520 (1984).

[114] T. Vachaspati, A. Vilenkin, Phys Rev. D31, 3035, (1985).

[115] R. Durrer, Nucl. Phys. B328, 238 (1989).

[116] R.L. Davis, Phys. Rev. D38, 3722 (1988).

[117] R.L. Davis, E.P.S. Shellard, Nucl. Phys. B323, 209 (1989).

[118] B. Carter, Ann. N.Y. Acad. Sci. 647, 758 (1991).

[119] M. James, L. Perivolaropoulos, T. Vachaspati, Nucl. Phys. B395, 534 (1993).

[120] B. Carter, Phys. Lett. B238, 166 (1990). 